\title{
Shared Aperture Antenna Technology for SAR: A Review of the Theory and Applications
}

\author{
Venkata Kishore Kothapudi* and Vijay Kumar \\ Photonics and Microwave division, School of Electronics Engineering, VIT University, Vellore, TN 632014 INDIA
}

Received 13 July 2016; Accepted 7 June 2017

\begin{abstract}
In order to use shared aperture antenna technology for Synthetic aperture radar, communication, Satellite communications, Electronic warfare and all radar applications (e.g., Space borne, aircraft telephony performances, antenna designs, wireless communications, airborne systems), studying the nature and characteristics of the shared aperture antenna technology is important. The purpose of this paper is to review and discuss various techniques aimed to develop the shared aperture antenna subsystem. This paper further focuses on the review of future implementation techniques and performance comparison along with their applications. This review serves as a comparative studies and reference beneficial for shared aperture antenna technology researchers and for future implementation of the technology. This review paper opens a corridor for researchers to perform future studies between different configurations and system models as a reference point for developing more powerful, flexible and efficient applications.
\end{abstract}

Keywords: Shared aperture antenna (SAA), Synthetic aperture radar (SAR), Space borne, Air borne and Wireless.

\section{Motivation and Background}

Shared aperture antennas are the becoming an active area of research. As we know the technology plays a pivotal role in the design and development of synthetic aperture radar systems. A good design can ensure that a system will satisfy key requirements in such topics as low mass, small volume, so as to reduce payload weight and size and thus the cost of the machine. So the task is to design and develop an effective SAA with less complexity that will support synthetic aperture radar applications. The above observational and budget constraints can be balanced by adopting antennas capable of working at dual polarization while sharing the same aperture, so as to reduce the overall size and weight of the system. As of now there is no systematic literature review for mapping shared aperture antenna technology.

The literature review aids researcher who is ambitious to contribute in this area, without investing time in doing a dedicated literature survey.

\subsection{Introduction to Synthetic aperture radar}

Since the advent of SAR technology by Carl A. Wiley, in 1954, it has been used as an important tool for earth observation from airborne as well as space-borne platforms. With the launch of Sea sat SAR in 1978, SAR has emerged as an immensely powerful operational research tool (Holtet et al., 1992; Katsaros and Brown, 1991) and tens of satellite placed in polar sun synchronous orbit. Importantly, SAR can provide quantitative measurement of earth surface features

\footnotetext{
*E-mail address: v.k.kothapudi@ieee.org

ISSN: 1791-2377 @ 2017 Eastern Macedonia and Thrace Institute of Technology. All rights reserved. doi:10.25103/iestr.103.07
}

and can also delineate the sub surface level geophysical parameters applying appropriate algorithms. It enables us to focus on a given research area for delineating the greater details with high to moderate resolution and high accuracy. SAR sensors can acquire data in single, double and quad polarization as per requirement of study, and meticulously used to geophysical parameters study using SAR interferometry and polarimetry. Antenna theory tells us that the antenna radiation pattern (i.e. a graphical representation of the intensity of the radiation as a function of the angle from the perpendicular line to the antenna plane) in the far field can be approximated to a Fourier transform of the physical shape of the antenna itself. Figure 1. shows an example of a rectangular antenna, which is the common shape of a SAR antenna, and its radiation pattern. The 3-D shape of the radiation pattern is shown in Figure 1. Which illustrates a typical imaging geometry of side-looking imaging radar. The rectangular SAR antenna is loaded either on an aircraft or on a satellite. As the platform moves along with the antenna, a stream of radar pulses is transmitted from the antenna. The platform's flight direction is called azimuth direction, and the transmission direction of radiated pulses in the main lobe is called range (or slant range) direction. The length of the footprint perpendicular to the azimuth direction is called swath width, and the angle of range from vertical is called look angle. Cumming and Wong, 2004 has provided details of SAR acquisition principle and imaging geometry. Foot print of SAR system depends on antenna design and characteristics and radar signal processing. There unique design of the antenna can help in acquiring images in multimode and multi polarization. Shared aperture antenna technology can be used to design a dual or triple band quad polarization and multi resolution SAR system. 


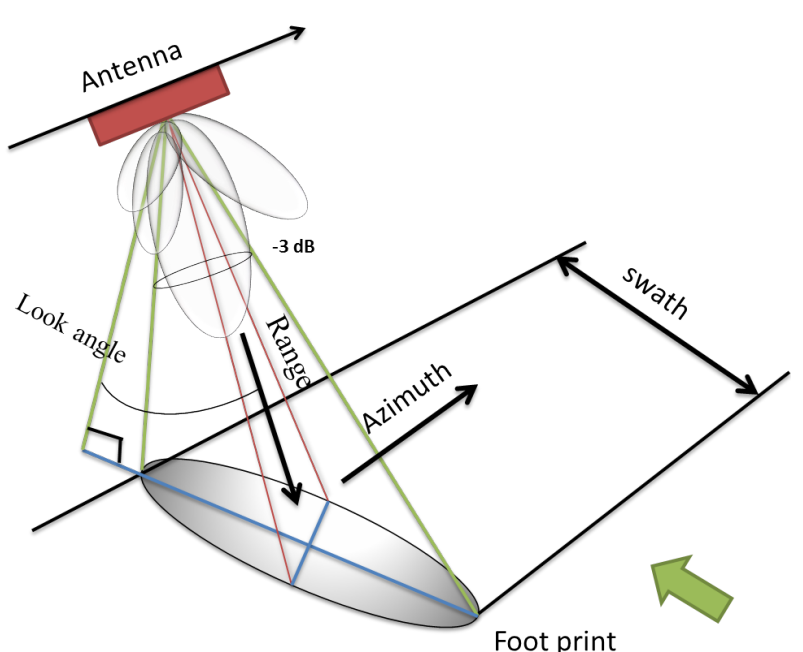

Fig 1. SAR technology

\subsection{SAA for SAR applications}

Antenna technology using shared aperture antenna (SAA) for dual band operation or using polarization diversity is of immense use in communication, especially in view of gradual increase in demand for communication channels. Such a technology also should help in reducing antenna cost, weight, as well as radar cross section (RCS). By the proper combination of element spacing, addressed in Table-8, amplitude weighting and progressive phase shift, the radiated energy can be concentrated into a main beam in particular scan angel given in Table-8, some minor lobes are called side lobes, are also formed. The input to the array is usually some form of transmission line i.e. coaxial cable. The overall characteristics of the array depend on the choice of the element and choice of the feeder network addressed in Table-9. Shared aperture antennas are the recent trend of antenna technology that integrate the usefulness of several antennas in to one aperture using dual/quad band multiple beamforming technology. The configuration of the SAA technology as shown in Figure 2. An aperture can be shared between systems in various ways. One way is to simply time multiplex the use of the aperture in Figure 2. To reduce the number of associated antenna systems by combining the functions of several systems in to one single aperture (A. T. Axness et al., 1995). Multi-functionality of the antenna system is a key issue in the case of mobile platforms performing simultaneous individual/multiple tasks, such as Radar applications, Synthetic aperture radar (SAR), VSAT, Space craft, 4G LTE, DCS, IEEE \&WIMAX and Wireless communication and electronic warfare etc. are addressed in Table-4 and Figure 6. Microstrip patch antenna is usually selected for its low profile and simplicity in fabrication. The trend in radars is to perform simultaneous multiple operations and measurements at dual/multiple frequencies ideally with dual polarization capability. Space borne synthetic aperture radar antennas have many special electrical requirements, such as operation at multiple frequencies with multiple polarization ability, with fairly wideband operation being required at these frequencies. They are also required to be electrically large, giving rise to issues such as low mass, easy and reliable deployability, and low cost and capability of high resolution imaging from airborne or space borne platforms.

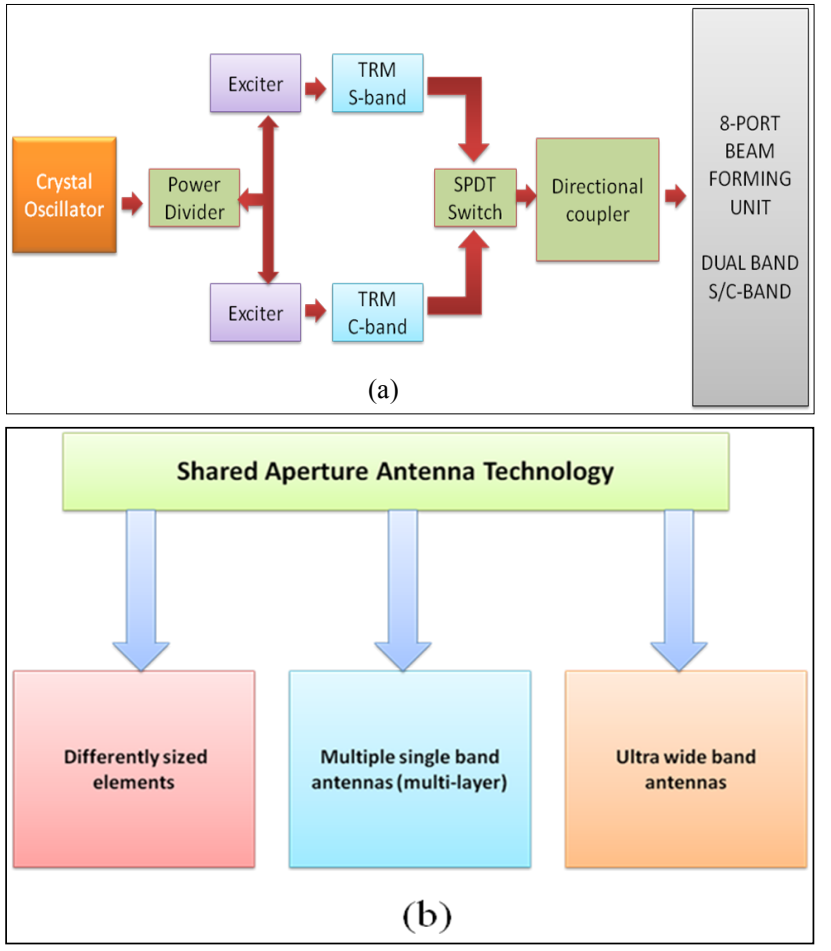

Fig. 2. (a) System configuration (b) SAA technology

A. Operating frequencies are usually widely separated (typically some combination of L/S-, S/C, L/X, $\mathrm{P} / \mathrm{Ku}$ or $\mathrm{P} / \mathrm{X}$-bands), requiring different array element spacings $(0.6 \lambda$ to $0.8 \lambda)$ to avoid grating lobes (particularly if scanning is required) and are preferred to reduce the number of antenna elements ultimately it leads to minimize cost mentioned in Table-5 in SLR.

B. Due to layout curtailment, a single microstrip feed substrate is limited to two independent feed networks (e.g., dual polarizations or dual frequencies). Thus, at least two/more substrate layers are required (given in Figure $6 \&$ Table 6) for dual polarization dual frequencies of operation.

C. Isolation between frequency bands are not likely to be met unless these feed networks are separated by a ground plane are addressed in Table- 11 .

This review firstly gives a motivation and back ground, introduction to shared aperture technology with related to synthetic aperture radar brief explanation about SAA. This is followed by highlighting the architectural infrastructure of SAA. Moreover, antenna design perspective based on the frequency range and the different methods have been explained to improve the cross polarization and isolation. The effects of SAA performance and analysis have also been reviewed. Finally, Shared aperture antenna technology have been used in different application ongoing researches.

\subsection{Research questions}

The main intention of this paper was to find and interpret the published literature related to shared aperture antenna technology for synthetic aperture radar applications to reduce payload weight and size and thus the cost of the machine. This is further detailed in the following research questions:

RQ1 How much activity was carried out in the last decade? 
RQ2 what research topics are being addressed?

RQ3What are the frequency bands and polarizations implemented with necessary applications.

RQ4 Software and Substrate used for design with spacing constrains for multi-layer or what are the different tools, technologies that were used?

RQ5 what are the choice of antenna elements (array) specified in SLR

RQ6 what are the type of feeding for antenna elements to improve perfect matching and the type of feeding network used for isolation and cross polarization improvement.
RQ7 what is the inter element spacing required and scan range used in SLR (systematic literature review).

RQ8 How the impedance matching, Return loss / VSWR / Reflection coefficient was improved in SLR

RQ9 How the isolation is achieved with different techniques.

RQ10 what are the configurations implemented for gain enhancement in SLR.

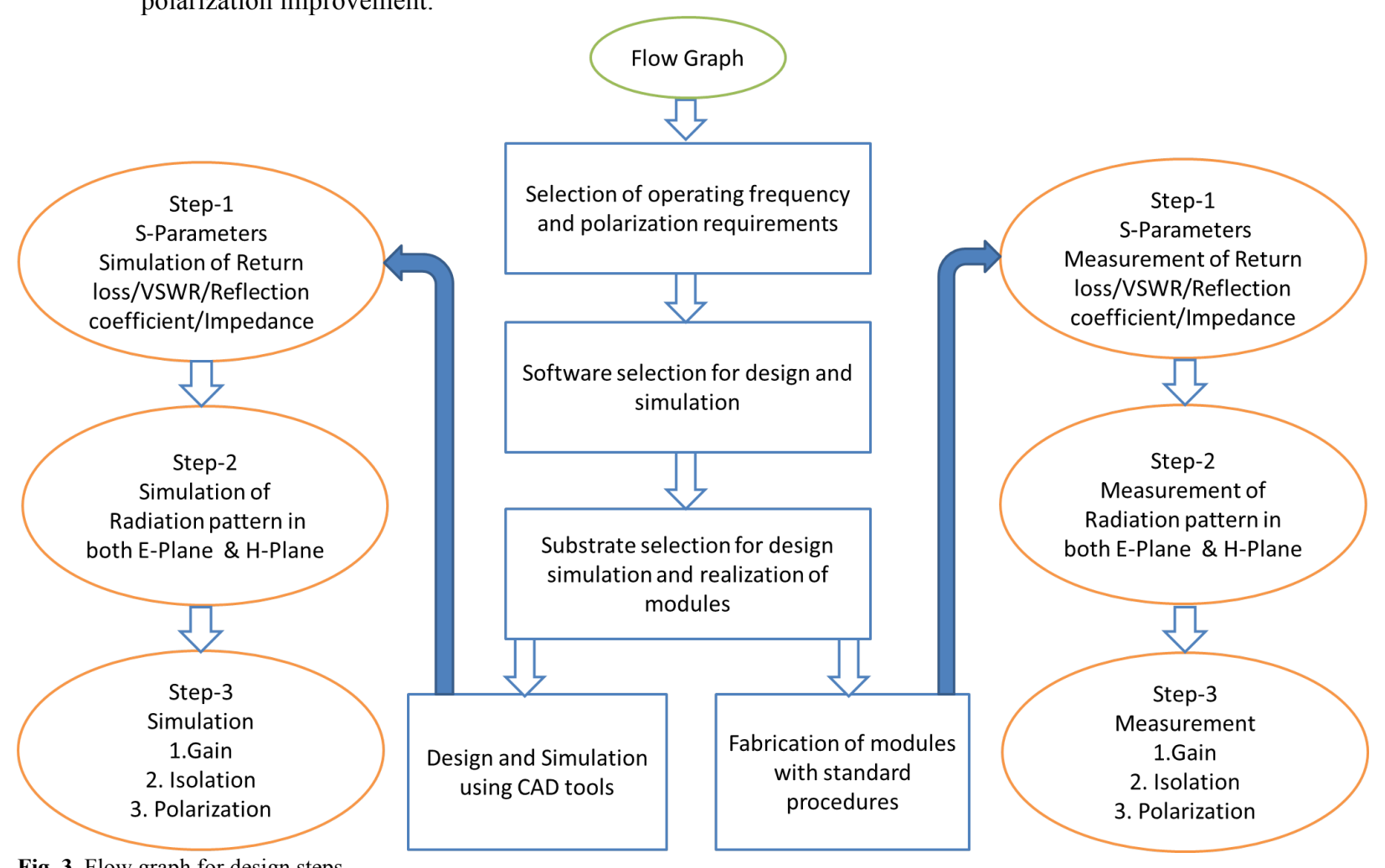

Fig. 3. Flow graph for design steps

\subsection{Related work}

There are no systematic literature reviews on Shared aperture antenna technology till now. Since then, the number of publications in the domain has not much increased and we focused on a systematic literature review from January 1996 to November 2015 from all locations with search strings only on shared aperture/shared aperture antenna.

\section{Search strategy}

We performed our search on scientific electronic databases which includes good impact factor conferences, journals and articles. Refer to Table 1 for a list of selected electronic databases.

Table 1. selected electronic databases

\begin{tabular}{l|l}
\hline Electronics database & URL \\
\hline IEEE & http://ieeexplore.ieee.org \\
PIERS & www.jpier.org \\
MOTL/Wiely & www.onlinelibrary.wiley.com \\
IET & www.theiet.org \\
\hline
\end{tabular}

\subsection{Search string}

Search string helps to get all results related to Shared aperture antenna. The reasons for searching with shared aperture and shared aperture antenna as keywords is to ensure all relevant papers are included. The search string used on all databases is: (shared aperture and shared aperture antenna).

\subsection{Inclusion criteria}

In order to include apropos publications in our review, we defined selection criteria and based on that we performed inclusion of published literature. We selected papers published in peer review journals, conferences and articles from $1 / 1 / 1996$ to $1 / 11 / 2015$. We selected papers that are relevant to our research questions. We excluded papers that are not related to shared aperture antenna technology. Table 2 shows our inclusion criteria.

Table 2. Inclusion criteria

\begin{tabular}{l}
\hline \multicolumn{1}{c}{ Inclusion } \\
\hline Publications from 1/1/1996 on Shared aperture antenna \\
technology related to Radar Applications. \\
Papers published in journals, conferences and articles. \\
\hline
\end{tabular}




\subsection{Roles and responsibilities}

Venkata Kishore Kothapudi (VIT University, research scholar): result classification and detailed analysis for various journals and papers from IEEE, PIERS and Wiely online (MOTL).

Vijay Kumar (VIT University, expert reviewer): assessment of classification and detailed analysis.

\subsection{Conference and journal selection process}

The process was conducted as follows:

1. The researchers perform the search on each database and save the references in bibliography files

2. The scholar reads all titles and abstracts and checks the inclusion and exclusion criteria for each entry

3. The scholar classifies the conferences and journals ccording to type, topic, and domain. The expert reviewer reassesses the classification and inclusion/exclusion of search results.

\subsection{Data analysis}

The data is analyzed to show:

1. The databases and number of query results

2. The publications are listed as per databases with respect to authorship, reference, date, publication type, type of content, topic of content and domain

3. The number of relevant publications per year

4. The graph that will show publication of journals and conferences, which are generated from the final results a detailed selection process performed on selected data bases.

\section{Results}

All results were ordered 'by relevance' as shown by the databases. From these results, we considered the first results of each database in our first repetition of the study. In total we reviewed 35 publications. The following acronyms are used to categorise the results in Tables 4.

Table 3. Number of papers per database

\begin{tabular}{l|l|l}
\hline Data base & Search date & Results \% \\
\hline IEEE & $1 / 10 / 2015$ & 63.33 \\
\hline
\end{tabular}

\begin{tabular}{l|l|l}
\hline PIERS & $1 / 10 / 2015$ & 6.66 \\
WIELY & $15 / 12 / 2015$ & 20 \\
IET & $1 / 10 / 15$ & 3.33 \\
INTECH OPEN SCI & $1 / 10 / 15$ & 3.33 \\
APL TECH DIGEST & $1 / 10 / 15$ & 3.33 \\
\hline
\end{tabular}

- Publication: The included publications classified as journals, conference papers and articles.

- Type: What kind of information was presented in the publication, e.g., date, publication, topic, application area. method.

- Topic: The exact intention and purpose of the publication.

- Application area: We classified publications into different areas, namely: Radar applications, Synthetic aperture radar (SAR), VSAT, Space craft, 4G LTE, DCS, IEEE \&WIMAX and Wireless. This will also ensure the publications are relevant to include in the review.

RQ1 How much work was carried out in last decade?

We plotted a number of relevant publications per databases in Figure 2, per publication type in Figure 3 and per year in Figure 4 . In the last decade, there was a noteworthy increase in number of publications compared with 2008 to 2015 that shows the significance of the review on shared aperture antenna technology. The first paper on shared aperture antenna technology with complete radar system configuration was published in 1996. Figure 4 shows numbers of papers published from 1996 to 2015 (Note: Search string only shared aperture/shared aperture antenna). We performed searches in October 2015 and December 2015 and all the papers had not been available by that time it might be the reason for less number of papers in 2015. The reviewed papers will help in building up a body of knowledge in shared aperture antenna technology for radar applications.

RQ2 what research topics are being addressed?

To know the research topics that are focused in shared aperture antenna technology, we have generated a table which are derived from topics and applications from year wise analysis are shown in Table 4. The research topics are broadly classified into Radar, Synthetic aperture radar, VSAT, Space craft, 4G LTE, DCS, IEEE \&WIMAX and Wireless application domains.

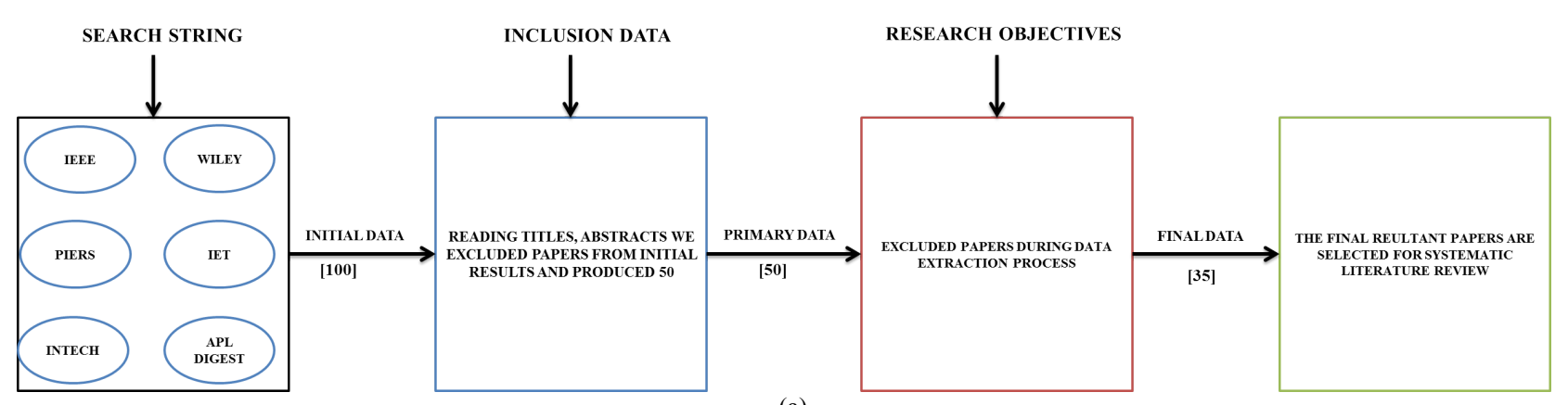

(a) 

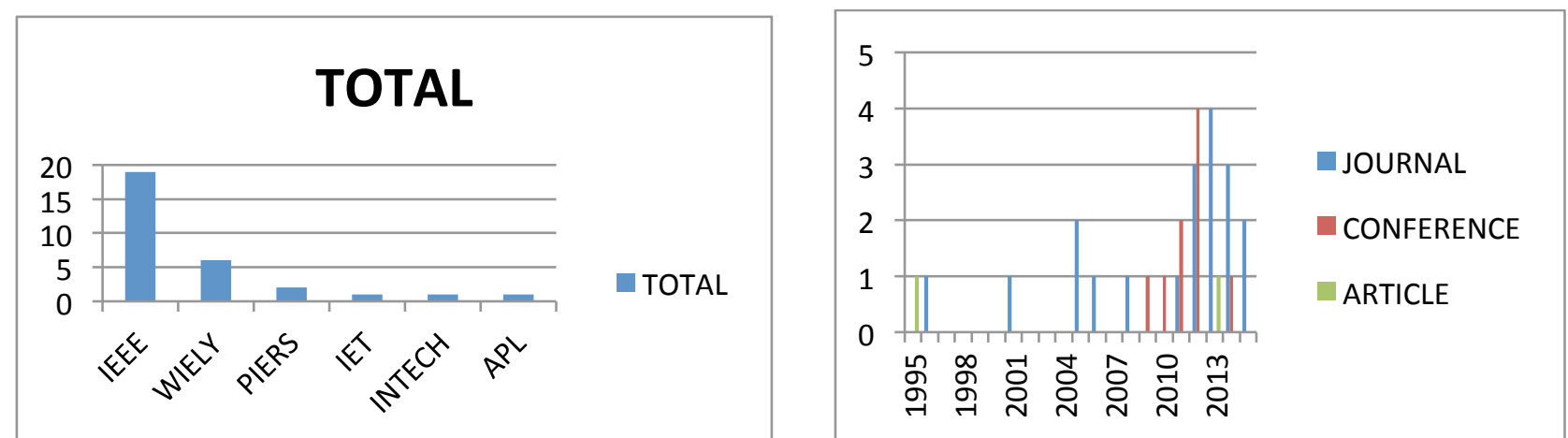

(b)

(c)

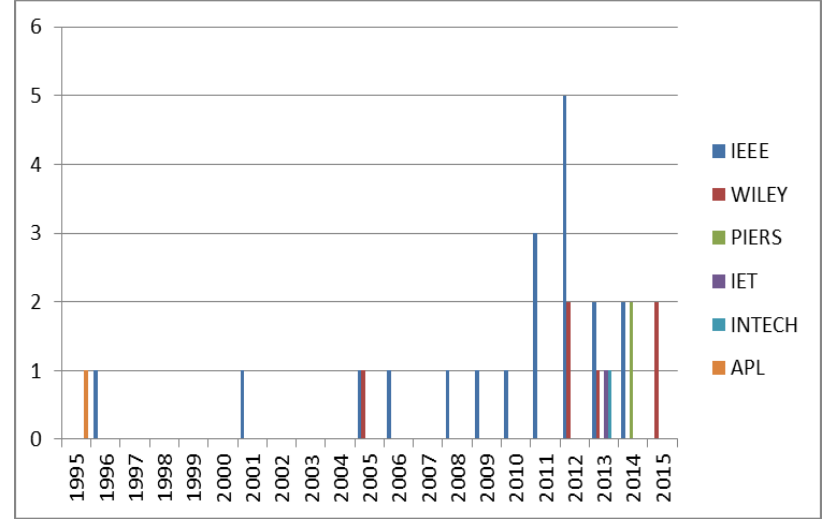

(d)

Fig. 4. (a) Selection process for review (b) Included results per database (c) Included results per publication type (d) Included results per year

RQ3 What are the frequency bands and polarizations implemented with necessary applications.

- Operating frequencies in $\mathrm{P}(\sim 0.4 \mathrm{GHz}), \mathrm{L}(\sim 1.3 \mathrm{GHz}), \mathrm{S}$ $(\sim 2.7 \mathrm{GHz}), \mathrm{C}(\sim 5.3 \mathrm{GHz}), \mathrm{X}(\sim 9.6 \mathrm{GHz})$, or $\mathrm{Ku}(\sim 17.2$ $\mathrm{GHz}$ ) band - the mostly used bands being L, C and $\mathrm{X}$.

- P-band most suited for biomass monitoring and hydrological mapping.

- $\quad$ S-band best suited for volumetric soil moisture.

- C-band covering the widest range (sea-ice, wave parameters by spectral analysis of image segments,
- Surface soil moisture, snow parameters, glaciers, ground water, etc.).

- X-band providing the best spatial resolution, thus best suited for surveillance.

- Ka-band specifically suited for snow that is semitransparent at lower frequencies.

- Several combinations of polarizations in transmission and reception possible to be implemented: $\mathrm{HH}, \mathrm{VV}$, $\mathrm{VV} / \mathrm{HH}, \mathrm{HH} / \mathrm{HV}$ and $\mathrm{VV} / \mathrm{VH}$.

- Wide range of applications for every frequency band, with variable effectiveness

Table 4. Specific topic and application area

\begin{tabular}{|c|c|c|c|c|c|}
\hline Author & Ref & Date & Publication & Topic & $\begin{array}{l}\text { Application } \\
\text { Area }\end{array}$ \\
\hline A. T. Axness et al. & [1] & 1995 & Article & An experimental shared aperture antenna development & COMM \&EW \\
\hline $\begin{array}{l}\text { Stephen D. Targonski } \\
\text { et al. }\end{array}$ & [2] & 1996 & Conference & A prototyne S A R array & SAR \\
\hline D.M. Pozar et al. & [3] & 1999 & Journal & $\begin{array}{c}\text { Dual-band and dual-polarization capability in a shared } \\
\text { aperture }\end{array}$ & SAR \\
\hline $\begin{array}{l}\text { Nemai C. Karmakar et } \\
\text { al. }\end{array}$ & [4] & 2001 & Journal & A photonic bandgap (PBG)-assisted shared-aperture & VSAT \\
\hline Vetharatnam, G et al. & [5] & 2005 & Journal & Combined feed network is proposed for ashared-aperture & SAR \\
\hline C.I. Coman et al. & [6] & 2006 & Journal & Differently sized radiating elements & RADAR \\
\hline Q.-X. Chu et al. & [7] & 2008 & Journal & Two wideband antennas sharing a common aperture & SAR \\
\hline M. Meng et al. & [8] & 2009 & Conference & A multilayer dual-band dual-polarized (DBDP) antenna & SAR \\
\hline Gao G et al. & [9] & 2010 & Conference & $\begin{array}{l}\text { Shared-aperture } \mathrm{Ku} / \mathrm{Ka} \text { bands microstrip array feeds of } \\
\text { parabolic cylindrical reflector antenna }\end{array}$ & $\begin{array}{l}\text { SPACE } \\
\text { CRAFT }\end{array}$ \\
\hline $\begin{array}{l}\text { Giuseppe Colangelo et } \\
\text { al. }\end{array}$ & {$[10]$} & 2011 & Conference & A novel Shared Aperture Dual Band Printed Antenna & SAR \\
\hline Shun-Shi Zhong et al. & [11] & 2011 & Conference & Tri-band dual-polarization (TBDP) sharec & SAR \\
\hline $\begin{array}{l}\text { Satyajit chakrabarti et } \\
\text { al. }\end{array}$ & [12] & 2011 & Journal & $\begin{array}{l}\text { Dual feed dual linearly polarized aperture coupled planar } \\
\text { microstrip patch antenna }\end{array}$ & SAR \\
\hline Zhong, S.-S et al. & {$[13]$} & 2012 & Joul & A tri-band dual-polarization (TBDP) shared-aperture & SAR \\
\hline Kong, L.-B et al. & [14] & 2012 & Journal & S/L-band dual-band dual-polarized shared-aperture array & SAR \\
\hline
\end{tabular}




\begin{tabular}{|c|c|c|c|c|c|}
\hline S.G. Zhou et al. & {$[15]$} & 2012 & Journal & $\begin{array}{c}\text { antenna } \\
\text { A shared-aperture dual-wideband dual-polarized planar } \\
\text { microstrip array }\end{array}$ & SAR \\
\hline Zhuo, S. G et al. & {$[16]$} & 2012 & Conference & $\begin{array}{l}\text { A design to integrate } \mathrm{P} \text {-band and } \mathrm{Ku} \text {-band antenna in a } \\
\text { shared compact planar aperture }\end{array}$ & SAR \\
\hline Zhuo, S. G et al. & {$[17]$} & 2012 & Conference & $\begin{array}{l}\text { a wideband dual polarization L/X-band shared aperture } \\
\text { antenna }\end{array}$ & SAR \\
\hline $\begin{array}{l}\text { Harender Singh Gusain } \\
\text { et al. }\end{array}$ & {$[18]$} & 2012 & Conference & $\begin{array}{c}\text { A circularly polarized }(\mathrm{CP}) \text { printed slot antenna } \\
\text { configuration on a shared aperture }\end{array}$ & SAR \\
\hline Zhuo, S. G et al. & {$[19]$} & 2012 & Conference & $\begin{array}{c}\text { the design of a dual-wideband dual linear polarization } \\
\text { shared aperture patch antenna }\end{array}$ & SAR \\
\hline Devendra Kumar & {$[20]$} & 2013 & Journal & a new type of wideband shared aperture dual band dual & SAR \\
\hline Sharma et al. & & & & $\begin{array}{l}\text { microstrip polarization patch antenna (MPA) operating at } \\
\text { L\&S band. }\end{array}$ & \\
\hline Shun-shi zhong et al. & {$[21]$} & 2013 & Journal & a shared-aperture MBDP SAR array & SAR \\
\hline A. B. Smolders et al. & {$[22]$} & 2013 & Journal & $\begin{array}{c}\text { array of aperture coupled microstrip antennas (ACMAs) } \\
\text { with corresponding feed network }\end{array}$ & SAR \\
\hline S.G. Zhou et al. & {$[23]$} & 2013 & Journal & A wideband, low-profile shared aperture antenna & SAR \\
\hline $\begin{array}{l}\text { Krishna Naishadham et } \\
\text { al. }\end{array}$ & {$[24]$} & 2013 & Journal & interleaved printed dipoles spaced to avoid grating lobes & SAR \\
\hline $\begin{array}{l}\text { M. Gulam Nabi Alsath } \\
\text { et al. }\end{array}$ & {$[25]$} & 2014 & Journal & $\begin{array}{l}\text { A modified planar inverted-F antenna (PIFA) and a "Y"- } \\
\text { shaped monopole sharing a common aperture }\end{array}$ & $\begin{array}{l}\text { 4G LTE, DCS, } \\
\text { IEEE } \\
\text { \&WIMAX }\end{array}$ \\
\hline Thomas Smith et al. & {$[26]$} & 2014 & Journal & $\begin{array}{l}\text { A Ka-band reflect array antenna with a frequency (FSS) } \\
\text { ground-plane }\end{array}$ & SAR \\
\hline Zhu Sun et al. & {$[27]$} & 2014 & Journal & $\begin{array}{l}\text { An } \mathrm{L} / \mathrm{C} \text { dual-band dual-polarized (DBDP) shared } \\
\text { aperture array }\end{array}$ & SAR \\
\hline Zeyang Tian et al. & {$[28]$} & 2014 & Conference & $\begin{array}{l}\text { three-dimensional (3D) substrate integrated waveguide } \\
\text { (SIW) technology }\end{array}$ & WIRELESS \\
\hline Zhou Shi-Gang et al. & {$[29]$} & 2015 & Journal & a dual-wideband dual-polarized planar shared aperture & SAR \\
\hline $\begin{array}{l}\text { Satyajit Chakrabarti et } \\
\text { al. }\end{array}$ & {$[30]$} & 2015 & Journal & $\begin{array}{l}\text { Shared aperture dual configuration antenna with dual } \\
\text { linearly/circularly polarized operation at } \mathrm{S} / \mathrm{Ka} \text {-band }\end{array}$ & SAR \\
\hline \multicolumn{6}{|c|}{$\begin{array}{l}\text { SAR- Synthetic Aperture Radar } \\
\text { DCS-Digital Cellular System } \\
\text { WIMAX- Worldwide Interoperability for Microwave Access } \\
\text { IEEE- Institute of Electrical and Electronics Engineers } \\
\text { LTE- Long Term Evolution }\end{array}$} \\
\hline
\end{tabular}

RQ4 Design Software tools and Substrate used for design with spacing constrains for multi-layer modeling.

The software used to model and simulate the antenna was designed using simulation tools addressed in Table. These tools can be used to calculate scattering parameters of antenna and radiation characteristics of the antenna mentioned in flow graph addressed in Figure 3.

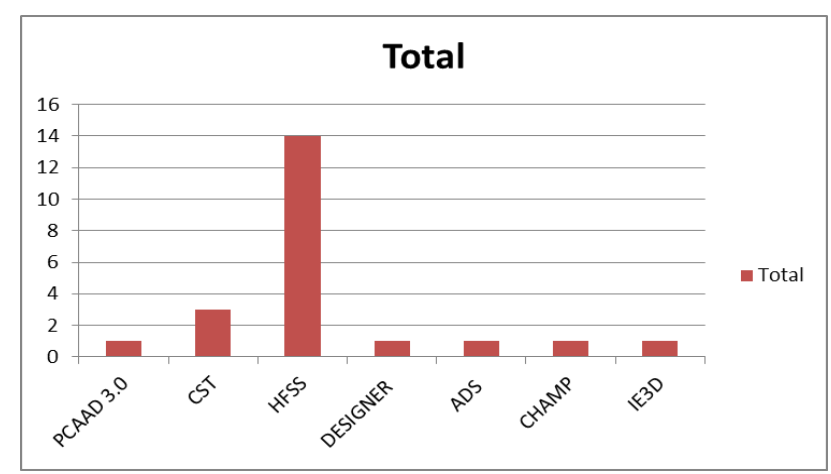

Fig. 5. Tools used for Simulation
The dielectric substrates used in SLR are FR4 Glass Epoxy, RO4003, Taconic TLC and RT Duroid $(5870 / 5880 / 6002)$ are addressed in Table 6

FR-4 glass epoxy is a popular and versatile high-pressure thermo set plastic laminate grade with good strength to weight ratios. With near zero water absorption, FR-4 is most commonly used as an electrical insulator possessing considerable mechanical strength.

RO4003 Series High Frequency Circuit Materials are glass reinforced hydrocarbon/ceramic laminates (Not PTFE) designed for performance sensitive, high volume commercial applications. RO4000 laminates are designed to offer superior high frequency performance and low cost circuit fabrication. The result is a low loss material which can be fabricated using standard epoxy/glass (FR4) processes.

Table 5. Operating frequencies and bands

\begin{tabular}{l|c|c|c|c|c|c|c|c|c|c}
\hline Author & $\begin{array}{c}\text { Frequency } \\
\text {-Band }\end{array}$ & P & L & S & C & X & KU & K & KA & W \\
\hline $\begin{array}{l}\text { A. T. Axness et al. } \\
\begin{array}{l}\text { Stephen D. Targonski et } \\
\text { al. }\end{array}\end{array}$ & C/X & & 1.25 & & 7 & $\mathbf{1 0}$ & & & & \\
D.M. Pozar et al. & L/X & & $\mathbf{1 . 2 1 - 1 . 2 9}$ & & & $\mathbf{9 . 5 - 9 . 8}$ & & & & \\
\hline
\end{tabular}




\begin{tabular}{|c|c|c|c|c|c|c|c|c|c|c|}
\hline Nemai C. Karmakar et & $\mathrm{S} / \mathrm{C}$ & & & 3.8 & 6.28 & & & & & \\
\hline Vetharatnam, G et al. & $\mathrm{L} / \mathrm{C}$ & & 1.24 & & 5.3 & & & & & \\
\hline C.I. Coman et al. & $\mathrm{C} / \mathrm{X}$ & & & & 8 & 9 & & & & \\
\hline Q.-X. Chu et al. & $\mathrm{P} / \mathrm{L}$ & $\begin{array}{c}110- \\
600\end{array}$ & $0.82-1.2$ & & & & & & & \\
\hline M. Meng et al. & $\mathrm{L} / \mathrm{C}$ & & 1.25 & & 5.3 & & & & & \\
\hline Gao G et al. & $\mathrm{Ku} / \mathrm{Ka}$ & & & & & & 13.6 & & 35.5 & \\
\hline $\begin{array}{l}\text { Giuseppe Colangelo et } \\
\text { al. }\end{array}$ & $\mathrm{X} / \mathrm{Ka}$ & & & & & & & & & \\
\hline Shun-Shi Zhong et al. & $\mathrm{L} / \mathrm{S} / \mathrm{X}$ & & 1.25 & 3.5 & & 10 & & & & \\
\hline $\begin{array}{l}\text { Satyajitchakrabarti et al. } \\
\text { Zhong S -S et al. }\end{array}$ & $\begin{array}{c}\mathrm{L} \\
\mathrm{L} / \mathrm{S} / \mathrm{X}\end{array}$ & & 1.4-1.65 & & & & & & & \\
\hline $\begin{array}{l}\text { Zhong, S.-S et al. } \\
\text { Kong, L.-B et al. }\end{array}$ & $\begin{array}{l}\mathrm{L} / \mathrm{S} / \mathrm{\lambda} \\
\mathrm{L} / \mathrm{S}\end{array}$ & & & & & & & & & \\
\hline S.G. Zhou et al. & $\mathrm{L} / \mathrm{X}$ & & $\begin{array}{c}1.126- \\
1.217\end{array}$ & & & $9-10.18$ & & & & \\
\hline Zhuo, S. G et al. & $\mathrm{L} / \mathrm{X}$ & $\begin{array}{c}325- \\
424\end{array}$ & & & & & $14-16$ & & & \\
\hline Zhuo, S. G et al. & $\mathrm{P} / \mathrm{Ku}$ & & $\begin{array}{l}1.126- \\
1.217\end{array}$ & & & $\begin{array}{c}9,9.6 \\
10\end{array}$ & & & & \\
\hline $\begin{array}{l}\text { Harender Singh Gusain } \\
\text { et al. }\end{array}$ & $\mathrm{S} / \mathrm{C} / \mathrm{X}$ & & & 3.5 & $4.5,7.5$ & 8.5 & & & & \\
\hline Zhuo, S. G et al. & $\mathrm{L} / \mathrm{X}$ & & $1.08-1.23$ & & & 8.6- & & & & \\
\hline $\begin{array}{l}\text { Devendra Kumar Sharma } \\
\text { et al. }\end{array}$ & $\mathrm{L} / \mathrm{S}$ & & 1.25 & 2.5 & & & & & & \\
\hline Shun-shizhong et al. & $\mathrm{L} / \mathrm{S} / \mathrm{X}$ & & 1.275 & 3 & & 9.6 & & & & \\
\hline A. B. Smolders et al. & $\mathrm{C}$ & & & & 4,8 & & & & & \\
\hline S.G. Zhou et al. & $\mathrm{P} / \mathrm{Ku}$ & $\begin{array}{c}330- \\
420\end{array}$ & & & & & $\begin{array}{c}14.27- \\
16.2\end{array}$ & & & \\
\hline $\begin{array}{l}\text { Krishna Naishadham et } \\
\text { al. }\end{array}$ & L & & $1-2$ & & & & & & & \\
\hline $\begin{array}{l}\text { M. GulamNabiAlsath et } \\
\text { al. }\end{array}$ & $\mathrm{L} / \mathrm{S}$ & & 1.8 & $\begin{array}{l}2.4, \\
3.5\end{array}$ & & & & & & \\
\hline Thomas Smith et al. & $\mathrm{L} / \mathrm{K} / \mathrm{Ka}$ & & $\begin{array}{l}1.525- \\
1.661\end{array}$ & & & & & $\begin{array}{l}19.7- \\
20.2\end{array}$ & $\begin{array}{c}29.5- \\
30\end{array}$ & \\
\hline Zhu Sun et al. & $\mathrm{L} / \mathrm{C}$ & & 1.25 & & 5.5 & & & & & 75 \\
\hline ZeyangTianet al. & $\mathrm{Ka} / \mathrm{W}$ & & & & & & & & 28 & \\
\hline Zhou Shi-Gang et al. & $\mathrm{L} / \mathrm{X}$ & & $1.07-1.24$ & & & 8.3- & & & & \\
\hline SatyajitChakrabarti et al. & & & & & & 10.3 & & & $27-29$ & v \\
\hline
\end{tabular}

RT/Duroid-6002 microwave material was the first low loss and low dielectric constant laminate to offer superior electrical and mechanical properties essential in designing complex microwave structures which are mechanically reliable and electrically stable. Applications particularly suited to the unique properties of RT/Duroid-6002 material include flat \& non-planar structures such as antennas, phased array antennas and beam forming networks.

RT/Duroid-5870 and 5880 glass microfiber reinforced PTFE composites are designed for exacting stripline and microstrip circuit applications. The dielectric constant of $\mathrm{RT} /$ Duroid 5870 and 5880 laminates is uniform from panel to panel and is constant over a wide frequency range. Its low dissipation factor extends the usefulness of RT/Duroid 5870 and 5880 laminates to $\mathrm{Ku}$-band and above.

Taconic TLY-5 substrates are specifically designed to meet the low cost objectives for newly emerging commercial $\mathrm{RF} /$ microwave applications. These materials exhibit excellent mechanical and thermal stability and cost less than traditional PTFE substrates.

RQ5 what are the choice of antenna elements (array) specified in SLR.
The typical configurations of the shared-aperture DBDP planar arrays include the perforated structure, the interlaced layout and the overlapped layout. The perforated structure mainly Includes perforated-patch/patch, ring/patch and cross-

Patch/patch. The interlaced layout includes interlace patch with dipole/slot and interlaced slot with slot/dipole etc. Both the perforated structure and interlaced layout are commonly adopted in space- or air-borne applications because of their low profile performance. On the other hand, the overlapped layout can provide further improvement in the bandwidths of dual bands, but with larger antenna profile. The different options for selecting lower and higher band elements are addressed in Table-9 in SLR.

RQ6 what are the type of feeding for antenna elements to improve perfect matching and the type of feeding network used for isolation and cross polarization improvement.

The feeding of individual elements has to be designed with respect to perfect impedance matching. The pair-wise anti phase feeding technique and differential feeding technique was implemented to reduce levels of cross 
polarization levels between the ports and bands mentioned in Table 8 and the cross polarization levels also given in Table-12 in SLR. The perturbation will also raise element cross polarization level; however, it can be suppressed by applying pair-wise anti-phase feeding method in the array scale.

Table 6. Substrate and Software used in SLR

\begin{tabular}{|c|c|c|c|c|c|c|}
\hline \multirow{2}{*}{$\begin{array}{c}\text { Author } \\
\text { A. T. Axness et al. }\end{array}$} & \multicolumn{2}{|c|}{ Frequency -Band } & \multirow{2}{*}{$\begin{array}{c}\text { SAA } \\
\text { Single } \\
\text { layer(1) }\end{array}$} & \multirow{2}{*}{$\begin{array}{c}\begin{array}{c}\text { Substrate } \\
\text { used }\end{array} \\
\text { RT-Duroid }\end{array}$} & \multirow{2}{*}{$\frac{\text { Foam used }}{\mathrm{Na}}$} & \multirow{2}{*}{$\begin{array}{c}\text { Software used } \\
\mathrm{Na}\end{array}$} \\
\hline & $\mathrm{C} / \mathrm{X}$ & WBDP & & & & \\
\hline Stephen D. Targonski et al. & $\mathrm{L} / \mathrm{X}$ & DBDP & Multi-layer(5) & RT-Duroid & Rohacell IG-51 & $\mathrm{Na}$ \\
\hline D.M. Pozar et al. & $\mathrm{L} / \mathrm{X}$ & DBDP & Multi-layer(5) & RT-Duroid & Rohacell IG-51 & $\begin{array}{c}\text { CAD TOOL PCAAD } \\
3.0\end{array}$ \\
\hline emai C. Karmakar et al. & $\mathrm{S} / \mathrm{C}$ & DBDP & Multi-layer(3) & RT-Duroid & Rohacell 51HF & $\mathrm{Na}$ \\
\hline Vetharatnam, $\mathrm{G}$ et al. & $\mathrm{L} / \mathrm{C}$ & DBDP & Multi-layer(5) & RT-Duroid & Rohacell 51HF & $\mathrm{Na}$ \\
\hline C.I. Coman et al. & $\mathrm{C} / \mathrm{X}$ & DBDP & $\begin{array}{l}\text { Single } \\
\text { layer(1) }\end{array}$ & RT-Duroid & $\mathrm{Na}$ & CST \\
\hline Q.-X. Chu et al. & $\mathrm{P} / \mathrm{L}$ & DBDP & $\begin{array}{l}\text { Single } \\
\text { layer(1) }\end{array}$ & RT-Duroid & $\mathrm{Na}$ & CST \\
\hline M. Meng et al. & $\mathrm{L} / \mathrm{C}$ & DBDP & Multi-layer(5) & FR4 & Air Honey comb & HFSS \\
\hline Gao G et al. & $\mathrm{Ku} / \mathrm{Ka}$ & DBDP & Multi-layer(4) & RT-Duroid & Rohacell 51HF & HFSS \\
\hline Giuseppe Colangelo et al. & $\mathrm{X} / \mathrm{Ka}$ & DBDP & Multi-layer(5) & RT-Duroid & Rohacell 51HF & $\mathrm{Na}$ \\
\hline Shun-Shi Zhong et al. & $\mathrm{L} / \mathrm{S} / \mathrm{X}$ & TBDP & Multi-layer(5) & RT-Duroid & Rohacell 51HF & HFSS- 10.0 \\
\hline Satyajit chakrabarti et al. & $\mathrm{L}$ & DBDP & $\begin{array}{l}\text { Single } \\
\text { layer(1) }\end{array}$ & RT-Duroid & $\mathrm{Na}$ & IE3D \\
\hline Zhong, S.-S et al. & $\mathrm{L} / \mathrm{S} / \mathrm{X}$ & TBDP & Multi-layer(5) & RT-Duroid & Rohacell $51 \mathrm{HF}$ & HFSS-10.0 \\
\hline Kong, L.-B et al. & $\mathrm{L} / \mathrm{S}$ & DBDP & Multi-layer(5) & RT-Duroid & Rohacell $51 \mathrm{HF}$ & HFSS \\
\hline S.G. Zhou et al. & $\mathrm{L} / \mathrm{X}$ & DBDP & Multi-layer(7) & RT-Duroid & Rohacell $51 \mathrm{HF}$ & HFSS-12.1 \\
\hline Zhuo, S. G et al. & $\mathrm{L} / \mathrm{X}$ & DBDP & Multi-layer(7) & FR4 & Rohacell 51HF & HFSS-12.1 \\
\hline Zhuo, S. G et al. & $\mathrm{P} / \mathrm{Ku}$ & DBDP & Multi-layer(7) & RT-Duroid & Rohacell 51HF & HFSS \\
\hline $\begin{array}{l}\text { Harender Singh Gusain et } \\
\text { al. }\end{array}$ & $\mathrm{S} / \mathrm{C} / \mathrm{X}$ & DBDP & $\begin{array}{l}\text { Single } \\
\text { layer(1) }\end{array}$ & RT-Duroid & $\mathrm{Na}$ & HFSS \\
\hline Zhuo, S. G et al. & $\mathrm{L} / \mathrm{X}$ & DBDP & Multi-layer(4) & $\begin{array}{c}\text { Arlon } \\
\text { DICLAD880, } \\
\text { Taconic TLY-5 }\end{array}$ & Rohacell 51HF & $\begin{array}{c}\text { ANSOFT DESIGNER } \\
\text { V-4.0 }\end{array}$ \\
\hline $\begin{array}{l}\text { Devendra Kumar Sharma et } \\
\text { al. }\end{array}$ & $\mathrm{L} / \mathrm{S}$ & DBDP & Multi-layer(9) & RT-Duroid & Rohacell 51HF & HFSS-10.0 \\
\hline Shun-shizhong et al. & $\mathrm{L} / \mathrm{S} / \mathrm{X}$ & DBDP & Multi-layer(5) & RT-Duroid & Rohacell 51HF & ADS MOMENTUM \\
\hline A. B. Smolders et al. & $\mathrm{C}$ & DBDP & $\begin{array}{c}\text { Multi- } \\
\text { layer(Na) }\end{array}$ & RT-Duroid & Rohacell 51HF & HFSS \\
\hline S.G. Zhou et al. & $\mathrm{P} / \mathrm{Ku}$ & DLP/SLP & Multi-layer(5) & FR4, TLY-5 & Rohacell $51 \mathrm{HF}$ & CST \\
\hline Krishna Naishadham et al. & $\mathrm{L}$ & DBDP & $\begin{array}{l}\text { Single } \\
\text { layer(1) }\end{array}$ & Paper substrate & $\mathrm{Na}$ & $\mathrm{Na}$ \\
\hline $\begin{array}{l}\text { M. Gulam Nabi Alsath et } \\
\text { al. }\end{array}$ & $\mathrm{L} / \mathrm{S}$ & TBDP & $\begin{array}{c}\text { Single } \\
\text { layer(1) }\end{array}$ & FR4 & $\mathrm{Na}$ & CHAMP \\
\hline Thomas Smith et al. & $\mathrm{L} / \mathrm{K} / \mathrm{Ka}$ & DBDP & Multi-layer(3) & RT-Duroid & Rohacell $51 \mathrm{HF}$ & HFSS-13.0 \\
\hline Zhu Sun et al. & $\mathrm{L} / \mathrm{C}$ & DBDP & $\begin{array}{l}\text { Multi- } \\
\text { layer(Na) }\end{array}$ & RT-Duroid & Rohacell 51HF & HFSS \\
\hline ZeyangTianet al. & $\mathrm{Ka} / \mathrm{W}$ & DBDP & $\begin{array}{l}\text { Single } \\
\text { layer(1) }\end{array}$ & RT-Duroid & Rohacell 51HF & $\mathrm{Na}$ \\
\hline Zhou Shi-Gang et al. & $\mathrm{L} / \mathrm{X}$ & DBDP & $\begin{array}{l}\text { Multi- } \\
\text { layer(Na) }\end{array}$ & RT-Duroid & $\begin{array}{l}\text { Plastic strips on } \\
\text { the } \\
\text { edge }\end{array}$ & $\mathrm{Na}$ \\
\hline SatyajitChakrabarti et al. & $\mathrm{S} / \mathrm{Ka}$ & DBDP & Parabolic & & & HFSS \\
\hline $\begin{array}{l}\text { Note : } \\
\text { CST- FDTD/FEM based Comp } \\
\text { HFSS-FDTD/FEM based High } \\
\text { IE3D- MoM based Integral Eq } \\
\text { ADS Momentum- MoM based }\end{array}$ & n 3 - Di & $\begin{array}{l}\text { oftware } \\
\text { ture sim } \\
\text { ional So }\end{array}$ & 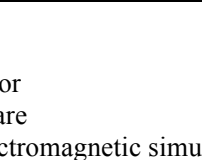 & & & \\
\hline
\end{tabular}

RQ7 what is the inter element spacing required and scan range used in SLR (systematic literature review).

Illumination taper:-

The side lobe requirement of $20 \mathrm{~dB}$ requires a suitable illumination taper.

Table 7. SLL and Taper efficiency

\begin{tabular}{l|l|l}
\hline Type & SLL & Taper efficiency \\
\hline Uniform & $-13 \mathrm{~dB}$ & $100 \%$ \\
Cosine & $-23 \mathrm{~dB}$ & $81 \%$ \\
Cosine square & $-32 \mathrm{~dB}$ & $66.7 \%$ \\
Cosine squared on pedestal & $-25.7 \mathrm{~dB}$ & $88 \%$ \\
Triangular & $-26.4 \mathrm{~dB}$ & $75 \%$ \\
Taylor & $-20 \mathrm{~dB}$ & $93.3 \%$ \\
\hline
\end{tabular}




\section{\begin{tabular}{l|l}
$-25 \mathrm{~dB}$ & $86.3 \%$ \\
\hline
\end{tabular}}

Taylor distribution has the advantage of high directivity with equal level close-in lower side lobes for a tapered envelope for the far-out side lobes.

Inter-element spacing:-

To keep the grating lobes from entering the visible region we have to select the inter-element spacing for an array according to the equation.

$\frac{d}{\lambda} \leq \frac{1}{1+\sin \theta}$

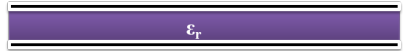

(a) Single Layer

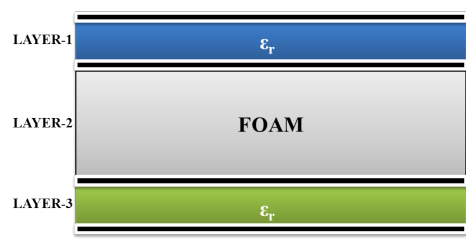

(b) 3-layer

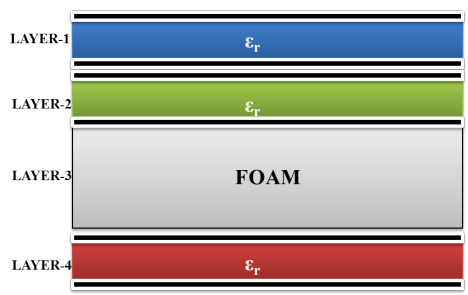

(c) 4-layer

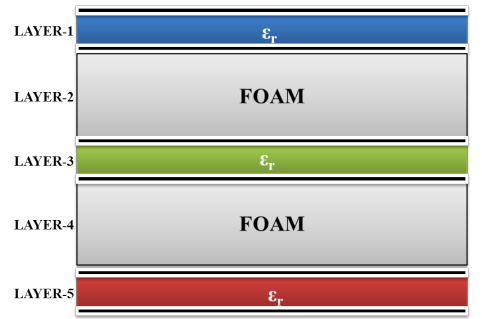

(d) 5-layer

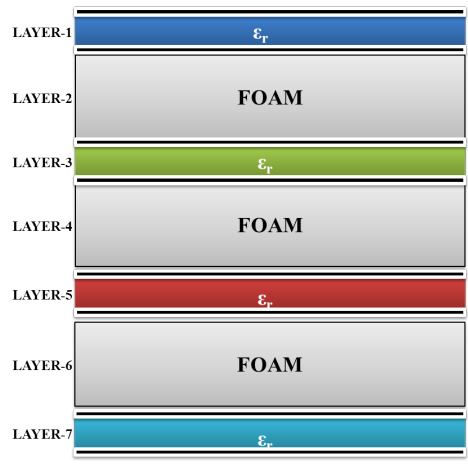

(e) 7-layer
Where $\theta$ is the scan angle

Table 8. Scan angle and Element spacing

\begin{tabular}{c|c}
\hline Scan angle & Element spacing \\
\hline 5 & $0.92 \lambda$ \\
10 & $0.85 \lambda$ \\
15 & $0.79 \lambda$ \\
20 & $0.75 \lambda$ \\
25 & $0.70 \lambda$ \\
30 & $0.66 \lambda$ \\
35 & $0.63 \lambda$ \\
40 & $0.60 \lambda$ \\
45 & $0.58 \lambda$ \\
50 & $0.56 \lambda$ \\
\hline
\end{tabular}

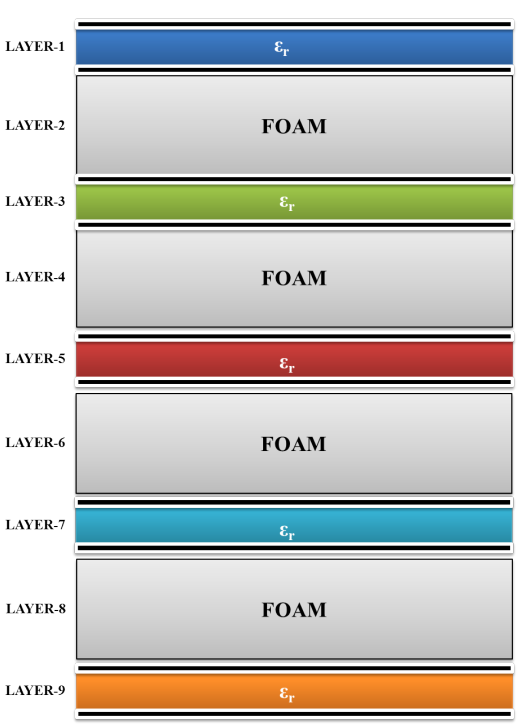

(f) 9-layer

Fig. 6. Single layer and Multi-layer configuration

RQ8 How the impedance matching, Return loss / VSWR / Reflection coefficient was improved in SLR.

Return loss is the difference in $\mathrm{dB}$ between the forward and reflected power measured at any given point in the RF system.

The SWR of the antenna is the mathematical relationship between the forward and reflected power measured at the feed point of the antenna. The simulated and measure return loss, VSWR at various frequencies of operation mentioned in Table 10 in SLR. Here again the relationship is linear, not logarithmic, and less reflected power (the better situation), the lower the value of SWR. With return loss, less reflected power means small number of $\mathrm{dBm}$ to be subtracted off, resulting in large return loss for better situations. Using our antenna example and the definition of the return loss given above, the return loss $(\mathrm{dB})$ generally measured at the input to the co-axial cable connected to the antenna.

To calculate return loss

1. Convert forward and reflected powers to $\mathrm{dB}$.

2. Subtract reflected power in $\mathrm{dB}$ from forward power.

Example 1:- The best case [VSWR=1:1]
Forward power $=1500$ watts $=61.76 \mathrm{dBm}$

Reflected power $=0$ watts $=0$

Return loss $=$ Forward power - Reflected power $=61.76 \mathrm{dBm}-$ Infinity $(\mathrm{dBm})$

$=$ Infinity $\mathrm{dB}$

Example 2:- The best case [VSWR $=1.05: 1]$

Forward power $=1500$ watts $=61.76 \mathrm{dBm}$

Reflected power $=1$ watts $=30 \mathrm{dBm}$

Return loss $=$ Forward power - Reflected power

Return loss $=$ Forward power - Reflected power

$$
=61.76 \mathrm{dBm}-30 \mathrm{dBm}
$$$$
=31.76 \mathrm{~dB}
$$

Example 3:- The best case [VSWR=1.92:1]

Forward power $=100$ watts $=50 \mathrm{dBm}$

Reflected power $=10$ watts $=40 \mathrm{dBm}$

Return loss $=$ Forward power - Reflected power

$$
=50 \mathrm{dBm}-40 \mathrm{dBm}
$$$$
=10 \mathrm{~dB}
$$ 
Example 4:- The best case [VSWR=infinity: 1]

Forward power $=100$ watts $=50 \mathrm{dBm}$

Reflected power $=100$ watts $=50 \mathrm{dBm}$

Return loss $=$ Forward power - Reflected power $=50 \mathrm{dBm}-50 \mathrm{dBm}$
$=0 \mathrm{~dB}$

This situation illustrates a dead short or completely open, with $100 \%$ forward power reflected back to the source. Reflection coefficient is the voltage form of return loss and mismatch loss is the amount of power lost due to reflection.

Table 9. Choice of Antenna element and feed network

\begin{tabular}{|c|c|c|c|c|c|c|c|}
\hline \multirow{2}{*}{$\begin{array}{l}\text { Author } \\
\text { A. T. Axness et al. }\end{array}$} & \multirow{2}{*}{$\begin{array}{l}\text { Date } \\
1995\end{array}$} & \multirow{2}{*}{$\begin{array}{l}\text { Publication } \\
\text { Article }\end{array}$} & \multicolumn{2}{|c|}{ Frequency-Band } & \multirow{2}{*}{$\begin{array}{l}\text { Antenna element } \\
\text { Tapered notch radiating } \\
\text { element }\end{array}$} & \multicolumn{2}{|l|}{$\begin{array}{l}\text { Element feed \& Feed } \\
\text { network }\end{array}$} \\
\hline & & & $\mathrm{C} / \mathrm{X}$ & WBDP & & Probe feed & A \\
\hline $\begin{array}{l}\text { Stephen D. Targonski et } \\
\text { al. }\end{array}$ & 1996 & Conference & $\mathrm{L} / \mathrm{X}$ & DBDP & Perforated patch/patch & Aperture coupled & A \\
\hline D.M. Pozar et al. & 1999 & Journal & $\mathrm{L} / \mathrm{X}$ & DBDP & Perforated patch/patch & Aperture coupled & $\mathrm{A}$ \\
\hline $\begin{array}{l}\text { Nemai C. Karmakar et } \\
\text { al. }\end{array}$ & 2001 & Journal & $\mathrm{S} / \mathrm{C}$ & DBDP & Rectangular patch & Aperture coupled & A \\
\hline Vetharatnam, G et al. & 2005 & Journal & $\mathrm{L} / \mathrm{C}$ & DBDP & $\begin{array}{l}\text { Square patch/square } \\
\text { perforated patch }\end{array}$ & Aperture coupled & A \\
\hline C.I. Coman et al. & 2006 & Journal & $\mathrm{C} / \mathrm{X}$ & DBDP & $\begin{array}{l}\text { Microstrip patch } \\
\text { antenna }\end{array}$ & Probe feed & B \\
\hline Q.-X. Chu et al. & 2008 & Journal & $\mathrm{P} / \mathrm{L}$ & DBDP & $\begin{array}{l}\text { Electrically loaded } \\
\text { monopole/electrically } \\
\text { loaded inverse L-shape } \\
\text { monopole }\end{array}$ & Microstripline feed & A \\
\hline M. Meng et al. & 2009 & Conference & $\mathrm{L} / \mathrm{C}$ & DBDP & $\begin{array}{l}\text { Perforated } \\
\text { patch/stacked patch }\end{array}$ & $\begin{array}{l}\text { Transmission line } \\
\text { feed/slot coupled }\end{array}$ & B \\
\hline Gao G et al. & 2010 & Conference & $\mathrm{Ku} / \mathrm{Ka}$ & DBDP & $\begin{array}{l}\text { Microstrip crossed } \\
\text { slots/patch }\end{array}$ & $\begin{array}{l}\text { Inversted microstrip } \\
\text { line/ microstrip line }\end{array}$ & A \\
\hline $\begin{array}{l}\text { Giuseppe Colangelo et } \\
\text { al. }\end{array}$ & 2011 & Conference & $\mathrm{X} / \mathrm{Ka}$ & DBDP & $\begin{array}{l}\text { Planar monopulse } \\
\text { printed antenna-Ka slot } \\
\text { array/X-slot array }\end{array}$ & Probe feed & A \\
\hline Shun-Shi Zhong et al. & 2011 & Conference & $\mathrm{L} / \mathrm{S} / \mathrm{X}$ & TBDP & $\begin{array}{l}\text { Microstrip } \\
\text { Dipole/patch/patch }\end{array}$ & $\begin{array}{l}\text { Microstrip line } \\
\text { feed/probe } \\
\text { feed/probe feed }\end{array}$ & B \\
\hline Satyajit chakrabarti et al. & 2011 & Journal & $\mathrm{L}$ & DBDP & Planar microstrip patch & Aperture coupled & A \\
\hline Zhong, S.-S et al. & 2012 & Journal & $\mathrm{L} / \mathrm{S} / \mathrm{X}$ & TBDP & $\begin{array}{l}\text { Microstrip } \\
\text { Dipole/patch/patch }\end{array}$ & $\begin{array}{l}\text { Microstrip line } \\
\text { feed/probe } \\
\text { feed/probe feed }\end{array}$ & B \\
\hline Kong, L.-B et al. & 2012 & Journal & $\mathrm{L} / \mathrm{S}$ & DBDP & $\begin{array}{l}\text { Stacked patch with } \\
\text { crossed slots }\end{array}$ & Aperture coupled & B \\
\hline S.G. Zhou et al. & 2012 & Journal & $\mathrm{L} / \mathrm{X}$ & DBDP & Microstrip array & Aperture coupled & $\mathrm{A}$ \\
\hline Zhuo, S. G et al. & 2012 & Conference & $\mathrm{L} / \mathrm{X}$ & DBDP & Patch/stacked patch & $\begin{array}{l}\text { L-probe } \\
\text { feed/Aperture } \\
\text { coupled }\end{array}$ & A \\
\hline Zhuo, S. G et al. & 2012 & Conference & $\mathrm{P} / \mathrm{Ku}$ & DBDP & $\begin{array}{l}\text { Perforated } \\
\text { patch/stacked patch }\end{array}$ & $\begin{array}{l}\text { Microstrip line } \\
\text { feed/proximity } \\
\text { coupled/aperture } \\
\text { coupled }\end{array}$ & A \\
\hline $\begin{array}{l}\text { Harender Singh Gusain } \\
\text { et al. }\end{array}$ & 2012 & Conference & $\mathrm{S} / \mathrm{C} / \mathrm{X}$ & DBDP & $\begin{array}{l}\text { Patch with dual slot } \\
\text { circular polarized } \\
\text { element }\end{array}$ & Microstrip line feed & A \\
\hline Zhuo, S. G et al. & 2012 & Conference & $\mathrm{L} / \mathrm{X}$ & DBDP & Microstrip patch & $\begin{array}{l}\text { proximity } \\
\text { coupled/aperture } \\
\text { coupled }\end{array}$ & $\mathrm{C}$ \\
\hline $\begin{array}{l}\text { Devendra Kumar } \\
\text { Sharma et al. }\end{array}$ & 2013 & Journal & $\mathrm{L} / \mathrm{S}$ & DBDP & $\begin{array}{l}\text { Square ring shaped } \\
\text { element/square shaped } \\
\text { patch }\end{array}$ & L-probe feed & A \\
\hline Shun-shi zhong et al. & 2013 & Journal & $\mathrm{L} / \mathrm{S} / \mathrm{X}$ & DBDP & L-dipole & $\begin{array}{l}\text { Microstrip line } \\
\text { feed/probe } \\
\text { feed/probe feed }\end{array}$ & $\mathrm{B}$ \\
\hline A. B. Smolders et al. & 2013 & Journal & $\mathrm{C}$ & DBDP & Microstrip patch & Aperture coupled & A \\
\hline S.G. Zhou et al. & 2013 & Journal & $\mathrm{P} / \mathrm{Ku}$ & DLP/SLP & Microstrip patch & Aperture coupled & $\mathrm{C}$ \\
\hline $\begin{array}{l}\text { Krishna Naishadham et } \\
\text { al. }\end{array}$ & 2013 & Journal & $\mathrm{L}$ & DBDP & $\begin{array}{l}\text { Microstrip folded } \\
\text { dipole }\end{array}$ & Microstrip line feed & A \\
\hline $\begin{array}{l}\text { M. Gulam Nabi Alsath et } \\
\text { al. }\end{array}$ & 2014 & Journal & $\mathrm{L} / \mathrm{S}$ & TBDP & $\begin{array}{l}\text { PIFA/ Y-shaped } \\
\text { monopole }\end{array}$ & Microstrip line feed & A \\
\hline Thomas Smith et al. & 2014 & Journal & $\mathrm{L} / \mathrm{K} / \mathrm{Ka}$ & DBDP & Patch/stacked patch & Probe feed & A \\
\hline Zhu Sun et al. & 2014 & Journal & $\mathrm{L} / \mathrm{C}$ & DBDP & Stacked patch & probe feed/ & B \\
\hline
\end{tabular}




\begin{tabular}{|c|c|c|c|c|c|c|c|}
\hline $\begin{array}{l}\text { Satyajit Chakrabarti et } \\
\text { al. }\end{array}$ & $\begin{array}{l}2014 \\
2015 \\
2015\end{array}$ & $\begin{array}{l}\text { Conference } \\
\text { Journal } \\
\text { Journal }\end{array}$ & $\begin{array}{l}\mathrm{Ka} / \mathrm{W} \\
\mathrm{L} / \mathrm{X} \\
\mathrm{S} / \mathrm{Ka}\end{array}$ & $\begin{array}{l}\text { DBDP } \\
\text { DBDP } \\
\text { DBDP }\end{array}$ & $\begin{array}{l}\text { Diploe/slot antenna } \\
\text { Microstrip patch } \\
\text { Parabolic reflector }\end{array}$ & $\begin{array}{l}\text { Microstrip line feed } \\
\text { Microstrip line feed } \\
\text { proximity } \\
\text { coupled/aperture } \\
\text { coupled } \\
\text { Cassegrain / } \\
\text { primary focus }\end{array}$ & $\begin{array}{l}\mathrm{A} \\
\mathrm{C}\end{array}$ \\
\hline $\begin{array}{rr}\text { Feeder network mentioned } \\
\text { A. } & \text { Corporate bina } \\
\text { B. } & \text { Pair-wise anti- } \\
\text { C. } & \text { Differential fee }\end{array}$ & $\begin{array}{l}\text { eder net } \\
\text { feeder } \\
\text { network }\end{array}$ & $\begin{array}{l}\text { ork } \\
\text { etwork }\end{array}$ & & & & & \\
\hline
\end{tabular}

Table 10. Source to load parameters with transmission line

loss is $3 \mathrm{~dB}$

\begin{tabular}{l|ll}
\hline & $\begin{array}{l}\text { Parameters } \\
\text { measured at } \\
\text { input }\end{array}$ & $\begin{array}{l}\text { Calculated } \\
\text { parameters at input to } \\
\text { the load }\end{array}$ \\
\hline $\begin{array}{l}\text { Forward } \\
\text { power }\end{array}$ & $100 \mathrm{~W}$ & $50.119 \mathrm{~W}$ \\
$\begin{array}{l}\text { Reflected } \\
\text { power }\end{array}$ & $10 \mathrm{~W}$ & $19.95 \mathrm{~W}$ \\
$\begin{array}{l}\text { Return loss } \\
\% \quad \text { power } \\
\text { reflected }\end{array}$ & $10 \mathrm{~dB}$ & $4 \mathrm{~dB}$ \\
$\begin{array}{l}\text { VSWR } \\
\text { Mismatch } \\
\text { loss }\end{array}$ & $10.92: 1$ & 39.81 \\
$\begin{array}{l}\text { Reflection } \\
\text { coefficient }\end{array}$ & $0.458 \mathrm{~dB}$ & $4.42: 1$ \\
\hline
\end{tabular}

RQ9 How the isolation is achieved with different techniques.

Isolation is sometimes referred to as on/off ratio. A minimum amount of isolation is required by the system designer to provide adequate rejection of unwanted signals in a given RF path. Isolation levels are range from $20 \mathrm{~dB}$ to $70 \mathrm{~dB}$ or more with value above $90 \mathrm{~dB}$ achievable but designs to achieve these levels are expense of other parameters. Feed methods, such as probe-feed, aperture-coupled, proximitycoupled, and L-shape feed are proposed to improve the key performances. Besides, balanced-feed and hybrid-feed are also implemented to enhance the port isolation, mentioned in Table 10. Important isolation measurement requirements are

a. Isolation between bands

b. Isolation between horizontal and vertical polarization ports

c. Isolation between co-pol and cross-pol levels.

Table 11. RL, Scan range, Spacing and Isolation

\begin{tabular}{|c|c|c|c|c|c|c|c|}
\hline Author & Frequ & $y$-Band & SAA & Return loss & Scan & & Isolation \\
\hline A. T. Axness et al. & $\mathrm{C} / \mathrm{X}$ & WBDP & $\begin{array}{l}\text { Single } \\
\text { layer(1) }\end{array}$ & $-10 \mathrm{~dB} /<2 / 50 \Omega$ & $\mathrm{Na}$ & $\mathrm{Na}$ & $\mathrm{Na}$ \\
\hline $\begin{array}{l}\text { Stephen D. Targonski et } \\
\text { al. }\end{array}$ & $\mathrm{L} / \mathrm{X}$ & DBDP & $\begin{array}{l}\text { Multi- } \\
\text { layer(5) }\end{array}$ & $-10 \mathrm{~dB} /<2 / 50 \Omega$ & $\pm 20^{0}-50^{0}$ & $0.6 \lambda-0.8 \lambda$ & 40 \\
\hline D.M. Pozar et al. & $\mathrm{L} / \mathrm{X}$ & DBDP & $\begin{array}{l}\text { Multi- } \\
\text { layer(5) }\end{array}$ & $-20 \mathrm{~dB} /<2 / 50 \Omega$ & $\pm 20^{0}-50^{0}$ & $0.6 \lambda-0.8 \lambda$ & 21 \\
\hline $\begin{array}{l}\text { Nemai C. Karmakar et } \\
\text { al. }\end{array}$ & $\mathrm{S} / \mathrm{C}$ & DBDP & $\begin{array}{l}\text { Multi- } \\
\text { layer(3) }\end{array}$ & $-15 \mathrm{~dB} /<2 / 50 \Omega$ & $\pm 20^{0}-50^{0}$ & $0.6 \lambda-0.8 \lambda$ & 30 \\
\hline Vetharatnam, G et al. & $\mathrm{L} / \mathrm{C}$ & DBDP & $\begin{array}{l}\text { Multi- } \\
\text { layer(5) }\end{array}$ & $-10 \mathrm{~dB} /<2 / 50 \Omega$ & $\pm 20^{0}-50^{0}$ & $0.6 \lambda-0.8 \lambda$ & 50 \\
\hline C.I. Coman et al. & $\mathrm{C} / \mathrm{X}$ & DBDP & $\begin{array}{l}\text { Single } \\
\text { layer(1) }\end{array}$ & $-10 \mathrm{~dB} /<2 / 50 \Omega$ & $\mathrm{Na}$ & $\mathrm{Na}$ & 25 \\
\hline Q.-X. Chu et al. & $\mathrm{P} / \mathrm{L}$ & DBDP & $\begin{array}{c}\text { Single } \\
\text { layer(1) }\end{array}$ & $-10 \mathrm{~dB} /<2 / 50 \Omega$ & $\mathrm{Na}$ & $\mathrm{Na}$ & $\mathrm{Na}$ \\
\hline M. Meng et al. & $\mathrm{L} / \mathrm{C}$ & DBDP & $\begin{array}{l}\text { Multi- } \\
\text { layer(5) }\end{array}$ & $-10 \mathrm{~dB} /<2 / 50 \Omega$ & $\pm 20^{0}-50^{0}$ & $0.6 \lambda-0.8 \lambda$ & $\mathrm{Na}$ \\
\hline Gao G et al. & $\mathrm{Ku} / \mathrm{Ka}$ & DBDP & $\begin{array}{c}\text { Multi- } \\
\text { layer(4) }\end{array}$ & $-10 \mathrm{~dB} /<2 / 50 \Omega$ & $\pm 20^{0}-50^{0}$ & $0.6 \lambda-0.8 \lambda$ & $\mathrm{Na}$ \\
\hline $\begin{array}{l}\text { Giuseppe Colangelo et } \\
\text { al. }\end{array}$ & $\mathrm{X} / \mathrm{Ka}$ & DBDP & $\begin{array}{l}\text { Multi- } \\
\text { layer(5) }\end{array}$ & $-10 \mathrm{~dB} /<2 / 50 \Omega$ & $\pm 20^{0}-50^{0}$ & $0.6 \lambda-0.8 \lambda$ & $\mathrm{Na}$ \\
\hline Shun-Shi Zhong et al. & $\mathrm{L} / \mathrm{S} / \mathrm{X}$ & TBDP & $\begin{array}{l}\text { Multi- } \\
\text { layer(5) }\end{array}$ & $-10 \mathrm{~dB} /<2 / 50 \Omega$ & $\pm 27^{0}$ & $0.6 \lambda-0.8 \lambda$ & $37,45,43$ \\
\hline Satyajit chakrabarti et al. & $\mathrm{L}$ & DBDP & $\begin{array}{l}\text { Single } \\
\text { layer(1) }\end{array}$ & $-10 \mathrm{~dB} /<2 / 50 \Omega$ & $\mathrm{Na}$ & $\mathrm{Na}$ & 30 \\
\hline Zhong, S.-S et al. & $\mathrm{L} / \mathrm{S} / \mathrm{X}$ & TBDP & $\begin{array}{l}\text { Multi- } \\
\text { layer(5) }\end{array}$ & $-10 \mathrm{~dB} /<2 / 50 \Omega$ & $\pm 20^{0}-50^{0}$ & $0.6 \lambda-0.8 \lambda$ & $37,45,43$ \\
\hline Kong, L.-B et al. & $\mathrm{L} / \mathrm{S}$ & DBDP & $\begin{array}{l}\text { Multi- } \\
\text { layer(5) }\end{array}$ & $-10 \mathrm{~dB} /<2 / 50 \Omega$ & $\pm 20^{0}-50^{0}$ & $0.6 \lambda-0.8 \lambda$ & 45 \\
\hline S.G. Zhou et al. & $\mathrm{L} / \mathrm{X}$ & DBDP & $\begin{array}{l}\text { Multi- } \\
\text { layer(7) }\end{array}$ & $-10 \mathrm{~dB} /<2 / 50 \Omega$ & $\pm 20^{0}-50^{0}$ & $0.6 \lambda-0.8 \lambda$ & 35 \\
\hline Zhuo, S. G et al. & $\mathrm{L} / \mathrm{X}$ & DBDP & Multi- & $-10 \mathrm{~dB} /<2 / 50 \Omega$ & $\pm 20^{0}-50^{0}$ & $0.6 \lambda-0.8 \lambda$ & 28 \\
\hline
\end{tabular}




\begin{tabular}{|c|c|c|c|c|c|c|c|}
\hline Zhuo, S. G et al. & $\mathrm{P} / \mathrm{Ku}$ & DBDP & $\begin{array}{l}\text { layer(7) } \\
\text { Multi- } \\
\text { layer(7) }\end{array}$ & $-10 \mathrm{~dB} /<2 / 50 \Omega$ & $\pm 20^{0}-50^{0}$ & $0.6 \lambda-0.8 \lambda$ & 20 \\
\hline $\begin{array}{l}\text { Harender Singh Gusain } \\
\text { et al. }\end{array}$ & $\mathrm{S} / \mathrm{C} / \mathrm{X}$ & DBDP & $\begin{array}{l}\text { Single } \\
\text { layer(1) }\end{array}$ & $-10 \mathrm{~dB} /<2 / 50 \Omega$ & $\mathrm{Na}$ & $\mathrm{Na}$ & $\mathrm{Na}$ \\
\hline Zhuo, S. G et al. & $\mathrm{L} / \mathrm{X}$ & DBDP & $\begin{array}{l}\text { Multi- } \\
\text { layer(4) }\end{array}$ & $-10 \mathrm{~dB} /<2 / 50 \Omega$ & $\pm 20^{0}-50^{0}$ & $0.6 \lambda-0.8 \lambda$ & 25,32 \\
\hline $\begin{array}{l}\text { Devendra Kumar } \\
\text { Sharma et al. }\end{array}$ & $\mathrm{L} / \mathrm{S}$ & DBDP & $\begin{array}{l}\text { Multi- } \\
\text { layer(9) }\end{array}$ & $-10 \mathrm{~dB} /<2 / 50 \Omega$ & $\pm 20^{0}-50^{0}$ & $0.6 \lambda-0.8 \lambda$ & \\
\hline Shun-shi zhong et al. & $\mathrm{L} / \mathrm{S} / \mathrm{X}$ & DBDP & $\begin{array}{l}\text { Multi- } \\
\text { layer(5) }\end{array}$ & $-10 \mathrm{~dB} /<2 / 50 \Omega$ & $\pm 20^{0}-50^{0}$ & $0.6 \lambda-0.8 \lambda$ & 12,15 \\
\hline A. B. Smolders et al. & $\mathrm{C}$ & DBDP & $\begin{array}{c}\text { Multi- } \\
\text { layer(Na) }\end{array}$ & $-10 \mathrm{~dB} /<2 / 50 \Omega$ & $\pm 20^{0}-50^{0}$ & $0.6 \lambda-0.8 \lambda$ & $37,45,43$ \\
\hline S.G. Zhou et al. & $\mathrm{P} / \mathrm{Ku}$ & DLP/SLP & $\begin{array}{l}\text { Multi- } \\
\text { layer(5) }\end{array}$ & $-10 \mathrm{~dB} /<2 / 50 \Omega$ & $\pm 20^{0}-50^{0}$ & $0.6 \lambda-0.8 \lambda$ & 26 \\
\hline $\begin{array}{l}\text { Krishna Naishadham et } \\
\text { al. }\end{array}$ & $\mathrm{L}$ & DBDP & $\begin{array}{l}\text { Single } \\
\text { layer(1) }\end{array}$ & $-10 \mathrm{~dB} /<2 / 50 \Omega$ & $\mathrm{Na}$ & $\mathrm{Na}$ & 17,25 \\
\hline $\begin{array}{l}\text { M. Gulam Nabi Alsath et } \\
\text { al. }\end{array}$ & $\mathrm{L} / \mathrm{S}$ & TBDP & $\begin{array}{l}\text { Single } \\
\text { layer(1) }\end{array}$ & $-10 \mathrm{~dB} /<2 / 50 \Omega$ & $\mathrm{Na}$ & $\mathrm{Na}$ & 15 \\
\hline Thomas Smith et al. & $\mathrm{L} / \mathrm{K} / \mathrm{Ka}$ & DBDP & $\begin{array}{l}\text { Multi- } \\
\text { layer(3) }\end{array}$ & $-10 \mathrm{~dB} /<2 / 50 \Omega$ & $\pm 20^{0}-50^{0}$ & $0.6 \lambda-0.8 \lambda$ & $\mathrm{Na}$ \\
\hline Zhu Sun et al. & $\mathrm{L} / \mathrm{C}$ & DBDP & $\begin{array}{c}\text { Multi- } \\
\text { layer(Na) }\end{array}$ & $-10 \mathrm{~dB} /<2 / 50 \Omega$ & $\pm 20^{0}-50^{0}$ & $0.6 \lambda-0.8 \lambda$ & 25 \\
\hline Zeyang Tian et al. & $\mathrm{Ka} / \mathrm{W}$ & DBDP & $\begin{array}{l}\text { Single } \\
\text { layer(1) }\end{array}$ & $-10 \mathrm{~dB} /<2 / 50 \Omega$ & $\mathrm{Na}$ & $\mathrm{Na}$ & $\mathrm{Na}$ \\
\hline Zhou Shi-Gang et al. & $\mathrm{L} / \mathrm{X}$ & DBDP & $\begin{array}{c}\text { Multi- } \\
\text { layer(Na) }\end{array}$ & $-10 \mathrm{~dB} /<2 / 50 \Omega$ & $\pm 20^{0}-50^{0}$ & $0.6 \lambda-0.8 \lambda$ & $22.5,29.6$ \\
\hline $\begin{array}{l}\text { Satyajit Chakrabarti et } \\
\text { al. }\end{array}$ & $\mathrm{S} / \mathrm{Ka}$ & DBDP & Parabolic & $-10 \mathrm{~dB} /<2 / 50 \Omega$ & $\pm 20^{0}-50^{0}$ & $0.6 \lambda-0.8 \lambda$ & 19,20 \\
\hline $\begin{array}{r}\text { Note :DBDP: Dual Band Du } \\
\text { TBDP: Triple Band D } \\
\text { DLP: Dual Linear Pol } \\
\text { SLP: Single Linear Pc } \\
\end{array}$ & $\begin{array}{l}\text { olarizat } \\
\text { Polariza } \\
\text { ation } \\
\text { zation } \\
\end{array}$ & & & & & & \\
\hline
\end{tabular}

Table 12. Gain, Side lobe level and Polartization

\begin{tabular}{|c|c|c|c|c|c|c|c|c|}
\hline \multirow{3}{*}{ Author } & \multicolumn{8}{|c|}{ Vertical and horizontal polarizations with different combinations } \\
\hline & \multicolumn{4}{|c|}{ E-plane } & \multicolumn{4}{|c|}{ H-plane } \\
\hline & Gain & SLL & Co-pol & X-pol & Gain & SLL & Co-pol & X-pol \\
\hline A. T. Axness et al. & $\mathrm{Na}$ & $>-10 \mathrm{~dB}$ & $0 \mathrm{~dB}$ & $\mathrm{Na}$ & $\mathrm{Na}$ & $>-10 \mathrm{~dB}$ & $0 \mathrm{~dB}$ & $\mathrm{Na}$ \\
\hline Stephen D. Targonski et al. & $\mathrm{Na}$ & $>-10 \mathrm{~dB}$ & $0 \mathrm{~dB}$ & $\mathrm{Na}$ & $\mathrm{Na}$ & $>-10 \mathrm{~dB}$ & $0 \mathrm{~dB}$ & $\mathrm{Na}$ \\
\hline D.M. Pozar et al. & $26 \mathrm{~dB}$ & $>-10 \mathrm{~dB}$ & $0 \mathrm{~dB}$ & -18 & $26 \mathrm{~dB}$ & $>-10 \mathrm{~dB}$ & $0 \mathrm{~dB}$ & $\mathrm{Na}$ \\
\hline Nemai C. Karmakar et al. & $5 \mathrm{~dB}$ & $>-10 \mathrm{~dB}$ & $0 \mathrm{~dB}$ & $\mathrm{Na}$ & $5 \mathrm{~dB}$ & $>-10 \mathrm{~dB}$ & $0 \mathrm{~dB}$ & $\mathrm{Na}$ \\
\hline Vetharatnam, G et al. & $\mathrm{Na}$ & $>-10 \mathrm{~dB}$ & $0 \mathrm{~dB}$ & $-40 \&-12$ & $\mathrm{Na}$ & $>-10 \mathrm{~dB}$ & $0 \mathrm{~dB}$ & $\mathrm{Na}$ \\
\hline C.I. Coman et al. & $\mathrm{Na}$ & $>-10 \mathrm{~dB}$ & $0 \mathrm{~dB}$ & $\mathrm{Na}$ & NA & $>-10 \mathrm{~dB}$ & $0 \mathrm{~dB}$ & $\mathrm{Na}$ \\
\hline Q.-X. Chu et al. & $5 \mathrm{dBi} / 21 \mathrm{dBi}$ & $>-10 \mathrm{~dB}$ & $0 \mathrm{~dB}$ & $\mathrm{Na}$ & $5 \mathrm{dBi} / 21 \mathrm{dBi}$ & $>-10 \mathrm{~dB}$ & $0 \mathrm{~dB}$ & $\mathrm{Na}$ \\
\hline M. Meng et al. & $9 \mathrm{dBi} / 9 \mathrm{dBi}$ & $>-10 \mathrm{~dB}$ & $0 \mathrm{~dB}$ & $-20 \&-30$ & $9 \mathrm{dBi} / 9 \mathrm{dBi}$ & $>-10 \mathrm{~dB}$ & $0 \mathrm{~dB}$ & $\mathrm{Na}$ \\
\hline Gao G et al. & $\mathrm{Na}$ & $-12.5 \mathrm{~dB}$ & $0 \mathrm{~dB}$ & -24 & $\mathrm{Na}$ & $-12.5 \mathrm{~dB}$ & $0 \mathrm{~dB}$ & $\mathrm{Na}$ \\
\hline Giuseppe Colangelo et al. & $\mathrm{Na}$ & $-13 \mathrm{~dB}$ & $0 \mathrm{~dB}$ & $\mathrm{Na}$ & $\mathrm{Na}$ & $-13 \mathrm{~dB}$ & $0 \mathrm{~dB}$ & $\mathrm{Na}$ \\
\hline Shun-Shi Zhong et al. & $\begin{array}{l}13.2- \\
22.2 \mathrm{dBi}\end{array}$ & $>-10 \mathrm{~dB}$ & $0 \mathrm{~dB}$ & -30 & $13.2-22.2 \mathrm{dBi}$ & $>-10 \mathrm{~dB}$ & $0 \mathrm{~dB}$ & $\mathrm{Na}$ \\
\hline Satyajit chakrabarti et al. & 14 & $-12 \mathrm{~dB}$ & $0 \mathrm{~dB}$ & -25 & 14 & $-12 \mathrm{~dB}$ & $0 \mathrm{~dB}$ & $\mathrm{Na}$ \\
\hline Zhong, S.-S et al. & $\begin{array}{l}13.2- \\
22.2 \mathrm{dBi}\end{array}$ & $>-10 \mathrm{~dB}$ & $0 \mathrm{~dB}$ & -30 & $13.2-22.2 \mathrm{dBi}$ & $>-10 \mathrm{~dB}$ & $0 \mathrm{~dB}$ & $\mathrm{Na}$ \\
\hline Kong, L.-B et al. & $\mathrm{Na}$ & $>-10 \mathrm{~dB}$ & $0 \mathrm{~dB}$ & $-30 \&-20$ & $\mathrm{Na}$ & $>-10 \mathrm{~dB}$ & $0 \mathrm{~dB}$ & $\begin{array}{l}-28 \&- \\
20\end{array}$ \\
\hline S.G. Zhou et al. & $10-25 \mathrm{dBi}$ & $>-10 \mathrm{~dB}$ & $0 \mathrm{~dB}$ & $-26 \&-24$ & $10-25 \mathrm{dBi}$ & $>-10 \mathrm{~dB}$ & $0 \mathrm{~dB}$ & $\mathrm{Na}$ \\
\hline Zhuo, S. G et al. & $5.4-24.4 \mathrm{dBi}$ & $>-10 \mathrm{~dB}$ & $0 \mathrm{~dB}$ & $\begin{array}{l}-18 \& \quad- \\
20.4\end{array}$ & $5.4-24.4 \mathrm{dBi}$ & $>-10 \mathrm{~dB}$ & $0 \mathrm{~dB}$ & $\mathrm{Na}$ \\
\hline Zhuo, S. G et al. & $5.8-21.2 \mathrm{dBi}$ & $>-10 \mathrm{~dB}$ & $0 \mathrm{~dB}$ & -20 & $5.8-21.2 \mathrm{dBi}$ & $>-10 \mathrm{~dB}$ & $0 \mathrm{~dB}$ & $\mathrm{Na}$ \\
\hline $\begin{array}{l}\text { Harender Singh Gusain et } \\
\text { al. }\end{array}$ & $3.58-4.1 \mathrm{dBi}$ & $>-10 \mathrm{~dB}$ & $0 \mathrm{~dB}$ & $\mathrm{Na}$ & $3.58-4.1 \mathrm{dBi}$ & $>-10 \mathrm{~dB}$ & $0 \mathrm{~dB}$ & $\mathrm{Na}$ \\
\hline Zhuo, S. G et al. & $\mathrm{Na}$ & $>-10 \mathrm{~dB}$ & $0 \mathrm{~dB}$ & $-20 \&-25$ & $\mathrm{Na}$ & $>-10 \mathrm{~dB}$ & $0 \mathrm{~dB}$ & $\mathrm{Na}$ \\
\hline $\begin{array}{l}\text { Devendra Kumar Sharma et } \\
\text { al. }\end{array}$ & $\begin{array}{l}6.94- \\
7.04 \mathrm{dBi}\end{array}$ & $>-10 \mathrm{~dB}$ & $0 \mathrm{~dB}$ & $12 \& 15$ & $6.94-7.04 \mathrm{dBi}$ & $>-10 \mathrm{~dB}$ & $0 \mathrm{~dB}$ & $12 \& 15$ \\
\hline Shun-shi zhong et al. & $\begin{array}{l}13.2- \\
22.19 \mathrm{dBi}\end{array}$ & $>-10 \mathrm{~dB}$ & $0 \mathrm{~dB}$ & -30 & $\begin{array}{l}13.2- \\
22.19 \mathrm{dBi}\end{array}$ & $>-10 \mathrm{~dB}$ & $0 \mathrm{~dB}$ & $\mathrm{Na}$ \\
\hline A. B. Smolders et al. & $5.1-5.4 \mathrm{dBi}$ & $>-10 \mathrm{~dB}$ & $0 \mathrm{~dB}$ & $\mathrm{Na}$ & $5.1-5.4 \mathrm{dBi}$ & $>-10 \mathrm{~dB}$ & $0 \mathrm{~dB}$ & $\mathrm{Na}$ \\
\hline S.G. Zhou et al. & $6.5-23.4 \mathrm{dBi}$ & $-11 \mathrm{~dB}$ & $0 \mathrm{~dB}$ & $-20 \&-26$ & $6.5-23.4 \mathrm{dBi}$ & $-11 \mathrm{~dB}$ & $0 \mathrm{~dB}$ & $\mathrm{Na}$ \\
\hline
\end{tabular}




\begin{tabular}{l|l|l|l|l|l|l|l|l}
\hline Krishna Naishadham et al. & $7-8 \mathrm{dBi}$ & $>-10 \mathrm{~dB}$ & $0 \mathrm{~dB}$ & -30 or -22 & $7-8 \mathrm{dBi}$ & $>-10 \mathrm{~dB}$ & $0 \mathrm{~dB}$ & -26 \\
M. Gulam Nabi Alsath et al. & $\mathrm{Na}$ & $>-10 \mathrm{~dB}$ & $0 \mathrm{~dB}$ & $\mathrm{Na}$ & $\mathrm{Na}$ & $>-10 \mathrm{~dB}$ & $0 \mathrm{~dB}$ & $\mathrm{Na}$ \\
Thomas Smith et al. & $36.4-$ & $-17 \mathrm{~dB}$ & $0 \mathrm{~dB}$ & $-23 \&-30$ & $36.4-38.5 \mathrm{dBi}$ & $-17 \mathrm{~dB}$ & $0 \mathrm{~dB}$ & $\mathrm{Na}$ \\
& $38.5 \mathrm{dBi}$ & & & & & & \\
& $12.9-$ & $>-10 \mathrm{~dB}$ & $0 \mathrm{~dB}$ & -28 & $12.9-26.8 \mathrm{dBi}$ & $>-10 \mathrm{~dB}$ & $0 \mathrm{~dB}$ & $\mathrm{Na}$ \\
Zhu Sun et al. & $26.8 \mathrm{dBi}$ & & & & & & & \\
& $4.8-11.7 \mathrm{dBi}$ & $>-10 \mathrm{~dB}$ & $0 \mathrm{~dB}$ & $\mathrm{Na}$ & $4.8-11.7 \mathrm{dBi}$ & $>-10 \mathrm{~dB}$ & $0 \mathrm{~dB}$ & $\mathrm{Na}$ \\
Zeyang Tian et al. & $6-23.2 \mathrm{dBi}$ & $>-10 \mathrm{~dB}$ & $0 \mathrm{~dB}$ & $-17 \&-20$ & $6-23.2 \mathrm{dBi}$ & $>-10 \mathrm{~dB}$ & $0 \mathrm{~dB}$ & $\mathrm{Na}$ \\
Zhou Shi-Gang et al. & $28.5-50 \mathrm{dBi}$ & $>-10 \mathrm{~dB}$ & $0 \mathrm{~dB}$ & -25 & $28.5-50 \mathrm{dBi}$ & $>-10 \mathrm{~dB}$ & $0 \mathrm{~dB}$ & $\mathrm{Na}$ \\
Satyajit Chakrabarti et al. & & & & & & & & \\
\end{tabular}

RQ10 what are the configurations implemented for gain enhancement in SLR.

The radiation pattern of the radar has to be carefully designed in order to obtain selectively backscattered signal as compared to interfering external influences like clutter, radio interference and scatter received through antenna side lobes. To achieve a high sensitivity and angular resolution, the antenna gain, addressed in Table 12 in SLR, should be large and beam width small. The main parameter determining the antenna gain and corresponding beam width is the area of the antenna which is called antenna aperture. An optimization of the aperture increases the sensitivity. Suppression of side lobes by tapering attenuates undesired signals but broadens the antenna beam.

The antenna of radar usually consists of either an array of individual elements to form a large antenna array or phase array and a large dish antenna. The aperture of phased array and the transmitter power is used to calculate the power aperture product which defines the sensitivity of the radar. The main antenna specifications which controls the design of phased array using shared aperture antenna technology for synthetic aperture radar are Gain, Beam width, Side lobe level and Beam scanning.

Array gain can be calculated by using

$\mathrm{G}_{\mathrm{A}}=10 \log$ (Number of elements)

+ Single element antenna gain

\section{Results}

This section provides a discussion of results and limitations of the study.

\subsection{Conclusions on the state of the art}

This Systematic Literature Review provides a study of shared aperture antenna for synthetic aperture radar applications. Though the results of reviews are reliable, they have potential threats to validity. The main intention of this review are the predispose in our selection of studies to be included, data extraction and synthesis. In order to reduce potential threats to validity, we define a research protocol, which contains research questions, inclusion criteria and research strategy.

In our search strategies, the main idea was to regain as much as possible of the available literature to avoid any predispose. Shared aperture antennas related to different radar and communication applications in order to cover all and avoid predispose. We searched for common terms (shared aperture and shared aperture antenna) and combined them in our search string, which decreases bias and increases search work.

The research protocol was developed by the first author and was reviewed by the second author, to ensure the review selection process and the search string was derived from research questions. To ensure correctness in data extraction, we summarized basic information which contains consistent and apropos data with respective to search string,inclusion and research questions. In order to mitigate reliability threat several researchers are involved in reviewing the included papers to achieve high validity of the study.

\subsection{Conclusions for a body of knowledge}

After analyzing the results of SLR, the body of knowledge has areas that represent shared aperture antenna which deals with Radar, Synthetic aperture radar, VSAT, Space craft, 4G LTE, DCS, IEEE \&WIMAX and Wireless application domains and various tools and technologies those are used in the study. This is illustrated in Figure 7

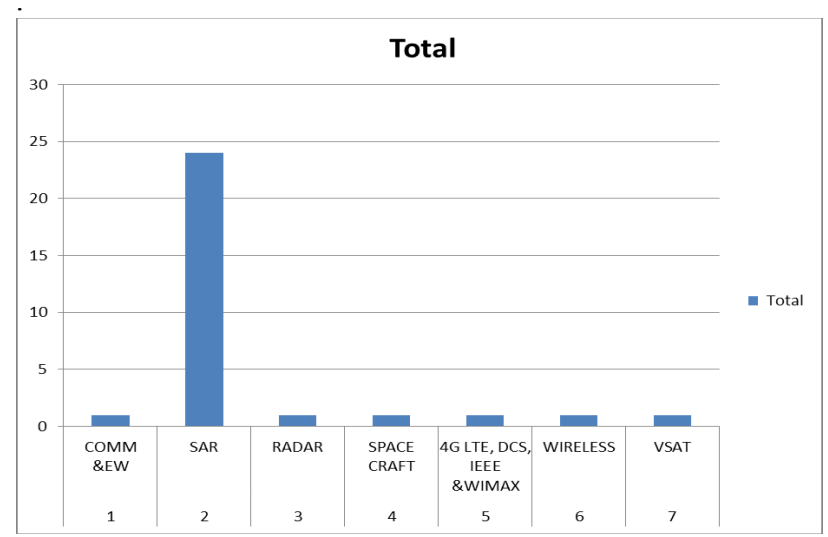

Fig. 7. Application domains for SAA technology

\section{Conclusion}

Shared aperture antennas has many advantages for future systems including reduced size and weight, easier integration and increased affordability when the cost is considered over multiple systems. The objective of this review was to consolidate existing research on shared aperture antenna technology and associated topics that allow for building up a body of knowledge. We considered 33 out of 100 reviewed publications significant with respect to research protocols, research question and categorized them according to the research area. On that basis, we provided tabulations for representing research areas, application domain, tools and technologies. We identified unexplored areas by synthesizing collected data, making those available for future research. We observed vast interests towards synthetic aperture radar and all radar applications. The field is still in its early stages and in order to mature, microwave and radar engineering researchers should come together by proposing a common research agenda. 


\section{Acknowledgment}

The work has been done at Microwave division, School of Electronics engineering (SENSE), VIT University, Vellore, and TN, India. All the assistance provided by the department and university administration to carry out this work is highly appreciated. The authors express their thanks to reviewers of this manuscript.authors would like to thanks the reviewers of this manuscript for their helpful comments.

This is an Open Access article distributed under the terms of the Creative Commons Attribution Licence

\section{References}

1. A. T. Axness, V. R. Coffman, A. B. Kopp, and W. O'Hawer, "Shared aperture technology development," John Hopkins APL Tech. Dig., vol.17, no. 3, pp. 285-294, 1997.

2. Stephen D. Targonski and David M. Pozar "An L/X dual-band dual polarized shared-aperture array for space borne SAR", IEEE Antennas and Propagation Society International Symposium, (1999). (Volume:4) pp.2306-2309

3. D.M. Pozar and S.D. Targonski, A shared-aperture dual-band dual polarized microstrip array, IEEE Trans Antennas Propag 49 (2001), 150-157.

4. Nemai C. Karmakar, Md. N. Mollah, Shantanu K Padhi, and Jeffrey S. Fu PBG-assisted shared-aperture dual-band aperture-coupled patch antenna for satellite communication Microwave and Optical Technology Letters, Jan. (2005). , 16(3), 289-292.

5. Vetharatnam, G., C. B. Kuan, and C. H. Teik, "Combined feed network for a shared-aperture dual-band dual-polarized array," IEEE Antennas Wirel. Propagat. Lett., Vol. 4, 297-299, 2005.

6. C.I. Coman, I. E. Lager and L. Light hart, "The Design of Shared Aperture Antenna Consisting of Differently Sized Elements", IEEE AP-54, February 2006.

7. Q.-X. Chu, M. Han-Qing, and H. Zheng, "Design of shared aperture wideband antennas considering band-notch and radiation pattern control," IEEE Trans. Antennas Propag., vol. 56, no. 11, pp. 3391-3395, Nov. 2008.

8. M. Meng, F. Zhang, X. Ding, K. Ding, and L. Li, "Design of a shared aperture dual-band dual- polarized microstrip antenna," in IEEE AP Symp. Dig., 2009, pp. 680-683.

9. Gao G. , Zhang, Y., Li, A., Zhao, J., \&Cheng, H., "Shared-aperture $\mathrm{Ku} / \mathrm{Ka}$ bands microstrip array feeds for parabolic cylindrical reflector", 2010 International Conference on Microwave and Millimeter Wave Technology (ICMMT), (2010). , 1028-1030.

10. Giuseppe Colangelo and Roberto Vitiello "Shared aperture dual band printed antenna" in IEEE International Conference on Electromagnetics in Advanced Applications (ICEAA), 2011, pp. 1092-1095.

11. Shun-Shi Zhong, Zhu Sun , Ling-Bing Kong Chu Gao , Wei Wang, and Mou-Ping Jin "Design of tbdp shared-aperture sar array", Asia-Pacific Microwave Conference Proceedings (APMC), 2011,pp. 159-162.

12. Satyajit chakrabarti "Development of shared aperture dual polarized microstrip antenna at L-band" ", IEEE Trans. Antennas Propagat., Jan. (2011)., 59(1).

13. Zhong, S.-S., Sun, Z., Kong, L.- B., Gao, C., Wang ,W., \& Jin, M.-P., "Tri-Band Dual Polarization Shared-Aperture Microstrip Array for SAR Applications", IEEE Trans. Antennas Propagat., Sep. (2012)., 60(9).

14. Kong, L.-B., Zhong, S.-S., \& Sun, Z., "Broadband microstrip element design of a DBDP shared-aperture SAR array", Microwave and Optical Technology Letters, Jan.(2012). , 54(1), $133-136$.

15. S.G. Zhou, T.H. Chio, and J. Lu, A shared-aperture dual-wideband dual-polarized stacked microstrip array, Microwave Opt TechnolLett 54 (2012), 486-491

16. Zhuo, S. G. and T. H. Chio, "A wideband, low profile p- and kuband dual polarized shared aperture antenna,"Proceedings of ISAP2012, 794-797, Nagoya, Japan, 2012.

17. Zhuo, S. G. and T. H. Chio, "Dual-wideband, dual-polarized shared aperture antenna with high isolation and low cross polarization," Proceedings of ISAP2012, 798-801, Nagoya, Japan, 2012.

18. S.G. Zhouand T.H. Chio, Dual-wideband, dual-polarized shared aperture antenna with high isolation and low cross-polarization, In: The 10th International Symposium on Antennas and Propagation, and EM theory, Xi'an, China, 2012.

19. Harender Singh Gusain, S.Raghavan and P.H.Rao "Shared aperture printed slot antenna"International Conference on Computing Communication \& Networking Technologies (ICCCNT), 2012, pp. 1-4.

20. Devendra Kumar Sharma, Sanjeev Kulshrestha, S. B. Chakrabarty, and Rajeev Jyoti Shared aperture dual band dual polarization microstrip patch antenna Microwave Opt Technol Lett 55 (2012), 917-922.

21. Shun-shi zhong,"Shared-aperture multi-band dual-polarized SAR microstrip array design", Intech.(2013).

22. A. B. Smolders, R. M. C. Mestrom, A. C. F. Reniers, and M. Geurts, "A shared aperture dual-frequency circularly polarized microstrip array antenna”, IEEE Antennas Wirel. Propagat. Lett., Vol. 12, 120-123, 2013.

23. Krishna Naishadham, Senior Member, IEEE, RongLin Li, Li Yang, Terrence Wu, Walker Hunsicker, and Manos Tentzeris, "A Shared-Aperture Dual-Band Planar Array With Self-Similar Printed Folded Dipoles", IEEE Trans. Antennas Propagat., Feb. (2013). , 61(2), pp. 606-613.

24. S.G. Zhou, P.K. Tan, and T.H. Chio, A wideband, low profile Pand $\mathrm{Ku}$-band shared aperture antenna with high isolation and low cross-polarization, IET Microwaves Antennas Propag 7 (2013), 223-229.

25. M. Gulam Nabi Alsath and Malathi Kanagasabai, "A sharedaperture multiservice antenna for automotive communications", IEEE Antennas Wirel. Propagat. Lett., Vol. 13, 1417-1420, 2014.

26. Thomas Smith, Ulrich Gothelf, Oleksiy S. Kim, and Olav Breinbjerg, "An FSS-Backed 20/30 GHz Circularly Polarized Reflect array for a Shared Aperture L- and Ka-Band Satellite Communication Antenna," IEEE Transactions on Antennas and Propagation, Feb. 2014, pp.661-668.

27. Zhu Sun, Karu P. Esselle, Shun-Shi Zhong, and Yingjie J. Guo Shared-Aperture Dual-Band Dual-Polarization Array Using Sandwiched Stacked Patch Progress In Electromagnetics Research C, Vol. 52, 183-195, 2014.

28. Zeyang Tian, Jun Ouyang, and Yu Long, "A shared aperture millimeter wave antenna using 3D SIW technology", PIERS Proceedings, Guangzhou, China, August 25-28, 2014.

29. Zhou Shi-Gang, Yang Jiang-Jun and Chio Tan-Huat Design of L/X-band shared aperture antenna array for SAR application Microwave Opt Technol Lett 57 (2015), 2197-2204.

30. Satyajit Chakrabarti, Development of shared aperture dual configuration antenna for S/Ka-band communication Microwave Opt Technol Lett 58 (2015), 139-145.

31. Wiley, C. A. (1954). Pulsed Doppler radar methods and apparatus. United States Patent, No. 3196436, Filed August 1954.

32. Katsaros KB and Brown, RA, (1991), Legacy of the Seasat Mission for the studies of the atmosphere and air-sea-ice interactions, Bull Am Met (Soc) 72(7), 967-981.

33. Cumming IG and Wong, FH (2004) Digital Synthesis of Synthetic Aperture Radar Data: Algorithms and Implementation, Artech House, Norwood. 